IZA DP No. 5107

Herausbildung erster Wesenszüge des Normalarbeitsverhältnisses in Deutschland

Michael J. Kendzia

August 2010

Forschungsinstitut zur Zukunft der Arbeit Institute for the Study of Labor 


\title{
Herausbildung erster Wesenszüge des Normalarbeitsverhältnisses in Deutschland
}

\author{
Michael J. Kendzia \\ IZA and University of Cologne
}

Discussion Paper No. 5107
August 2010

IZA

P.O. Box 7240

53072 Bonn

Germany

Phone: +49-228-3894-0

Fax: +49-228-3894-180

E-mail: iza@iza.org

\begin{abstract}
Any opinions expressed here are those of the author(s) and not those of IZA. Research published in this series may include views on policy, but the institute itself takes no institutional policy positions.

The Institute for the Study of Labor (IZA) in Bonn is a local and virtual international research center and a place of communication between science, politics and business. IZA is an independent nonprofit organization supported by Deutsche Post Foundation. The center is associated with the University of Bonn and offers a stimulating research environment through its international network, workshops and conferences, data service, project support, research visits and doctoral program. IZA engages in (i) original and internationally competitive research in all fields of labor economics, (ii) development of policy concepts, and (iii) dissemination of research results and concepts to the interested public.
\end{abstract}

IZA Discussion Papers often represent preliminary work and are circulated to encourage discussion. Citation of such a paper should account for its provisional character. A revised version may be available directly from the author. 


\section{ABSTRACT}

\section{Herausbildung erster Wesenszüge des Normalarbeitsverhältnisses in Deutschland}

The paper provides a historical overview of the development of the initial characteristics of the standard employment contract in Germany from the middle of the 19th century until the beginning of World War I. The beginning of the welfare state in Germany took place during the $1880 \mathrm{~s}$ on the basis of dependent employment. For the analysis of this type of employment, the model of the standard employment contract is used, and the specific factors which determined the rise of the standard employment contract are examined. In the 19th century, wage labour became more and more common and finally displaced the pre-modern allocation of work. After being freed from feudal dependence, the familiar social question arose. In this context, the main driving forces as well as different approaches in solving this question are surveyed. State regulations were implemented to protect the workforce against exploitation by industrial entrepreneurs. Later on, a social insurance system was created to protect workers against basic risks. The paper finds that prior to the establishment of the social insurance system, a number of insurance funds had existed which were financed by worker contributions. Moreover, these insurance funds already contained the essential structural elements of the later Bismarckian welfare system. The paper concludes that at the end of the observed period, dependent, full-time employment subject to social insurance contributions can be identified.

JEL Classification: J50, J40

Keywords: Abhängiges Beschäftigungssystem, Normalarbeitsverhältnis, Soziale Frage, Soziale Sicherung, Bismarck, Sozialversicherungsgesetzgebung

Corresponding author:

Michael J. Kendzia

IZA

P.O. Box 7240

D-53072 Bonn

Germany

E-mail: kendzia@iza.org 


\section{Herausbildung erster Wesenszüge des Normalarbeitsverhältnisses in Deutschland}

Michael J. Kendzia

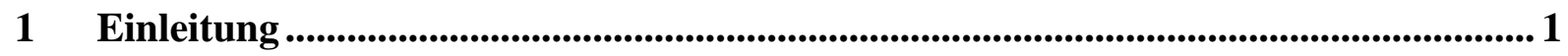

2 Durchsetzung der Lohnarbeit bei der Organisation gesellschaftlicher Arbeit .......... 2

2.1 Allgemeine Entwicklung der Beschäftigung bis 1914 ........................................ 2

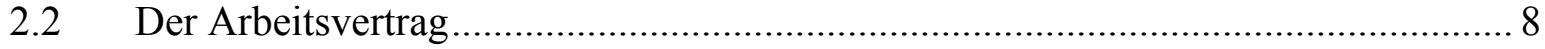

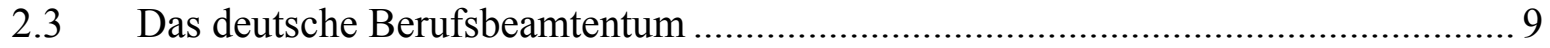

3 Entstehung der Sozialen Frage im 19. Jahrhundert .................................................... 13

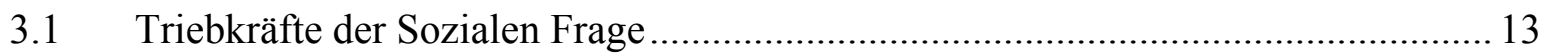

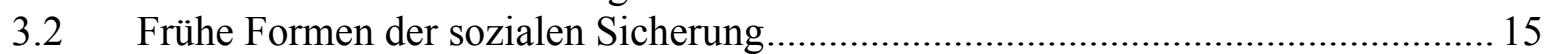

3.3 Unternehmerische und andere nicht-staatliche Initiativen ................................. 16

4 Institutionalisierung der Sozialversicherungssysteme durch Bismarck .................... 20

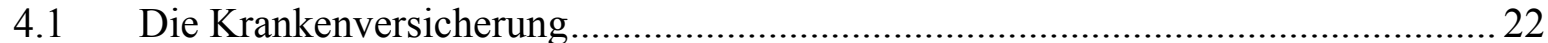

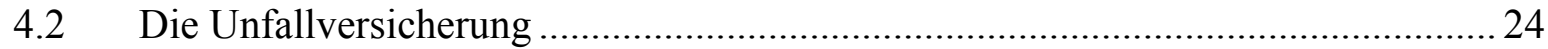

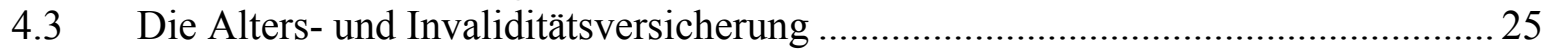

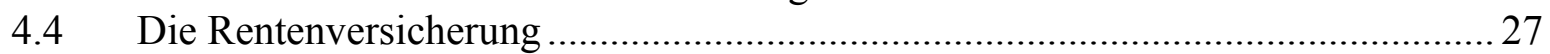

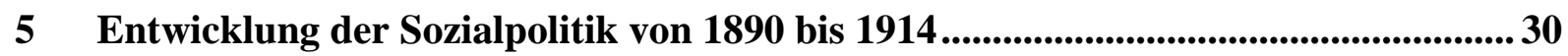

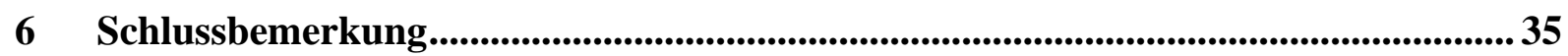




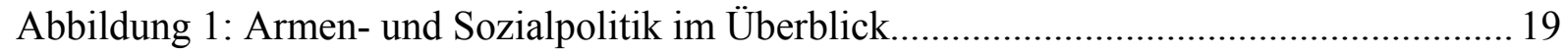

Abbildung 2: Berufsgruppen nach Berufszählungen ......................................................... 32

Abbildung 3: Arbeitszeit, Entlohnung und Arbeitslosigkeit 1820 bis 1913 ........................... 33 
Tabelle 1: Arbeitszeit in Deutschland von 1800 bis 1914

Tabelle 2: Indexziffern der Lebenshaltungskosten, der Nominal- und Reallöhne in

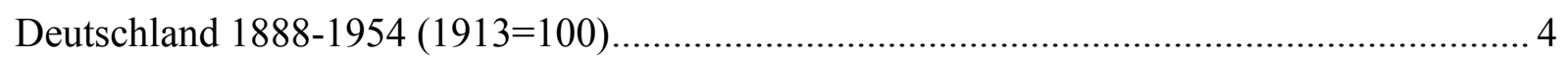

Tabelle 3: Beschäftigte in den Sektoren der Wirtschaft (1800-1913) ..................................... 6

Tabelle 4: Die Beschäftigten nach Wirtschaftsbereichen 1846-1913 (in Tausend).................. 6

Tabelle 5: Beispielrechnung der jährlichen Beiträge für die einzelnen Versicherungen .......... 26

Tabelle 6: Mitgliederzahl der Sozialversicherung von 1890 bis 1913 (in Millionen) ............. 28

Tabelle 7: Gesamtbeiträge zur Sozialversicherung (in Millionen Mark) 1887-1913 .............. 28 


\section{Einleitung}

Der Sozialstaat in Deutschland wurde in den 80er Jahren des 19. Jahrhunderts auf der Basis der Erwerbsarbeit errichtet. Er finanziert sich seitdem überwiegend über Beiträge der Arbeiter und Arbeitgeber - und nicht über Steuern oder andere Quellen (Kocka 2005: 201-205). Ein Modell für ein abhängiges Beschäftigungsverhältnis bildet seit geraumer Zeit das so genannte Normalarbeitsverhältnis. Unter dem Normalarbeitsverhältnis ist eine unbefristete und sozialversicherungspflichtige Erwerbsarbeit aufzufassen, die in Vollzeit betrieben wird. Als Namensgeber des Begriffes „Normalarbeitsverhältnis“ gilt Mückenberger (Mückenberger 1985b). Eine stärkere Ausdifferenzierung der Arbeits- und Lebensformen weicht das Fundament des lohn-, beitrags- und leistungsbezogenen Systems der sozialen Sicherung erkennbar auf (Butterwegge 2001: 54, Eichhorst et al. 2010). Sogar von einer „Erosion des Normalarbeitsverhältnisses“ (Wüstner 2006: 37) ist die Rede.

Der vorliegende Artikel will an vorangegangene Überlegungen anknüpfen und untersuchen (Pierenkemper/Kendzia 2010), welche konkreten Einflussfaktoren auf die Entstehung des Normalarbeitsverhältnisses einwirkten. Das Forschungsinteresse konzentriert sich dabei auf einen möglichen Beitrag des Berufsbeamtentums für die Herausbildung erster Merkmale des Normalarbeitsverhältnisses. Denn bisher ist unklar, welchen Einfluss das Berufsbeamtenverhältnis auf die Entstehung des Normalarbeitsverhältnisses hatte. Den theoretischen Rahmen der Rekonstruktion bietet ein polit-ökonomischer Ansatz, der sich im Wesentlichen mit der Aufteilung der Ressourcen auf die zentralen Akteure innerhalb einer Gesellschaft befasst.

Ausgehend von der Durchsetzung der Lohnarbeit bei der Organisation gesellschaftlicher Arbeit innerhalb des 19. Jahrhunderts in Deutschland, soll den Entstehungsgründen für die Soziale Frage nachgegangen werden. Hierzu werden neben verschiedenen Triebkräften der Entwicklung auch unternehmerische und andere nicht-staatliche Initiativen aufgezeigt, die sich noch vor der Einführung der Bismarckschen Sozialversicherungen mit der Absicherung der Lebensrisiken der entstandenen (Lohn-) Arbeiterschaft befassten. Daran anschließend, wird auf die staatlichen Lösungsansätze der Sozialen Frage eingegangen und die genaueren Gründe sowie die nähere Ausgestaltung der Bismarckschen Sozialgesetzgebung erläutert und einer polit-ökonomischen Analyse unterzogen. Abschließend werden weitere Arbeiterschutzbestimmungen behandelt, bevor mit dem Ausbruch des Ersten Weltkrieges und der Identifikation erster Wesenszüge des Normalarbeitsverhältnisses das vorläufige Ende der Untersuchung zum Normalarbeitsverhältnis erreicht wird. 


\section{Durchsetzung der Lohnarbeit bei der Organisation gesellschaftlicher Arbeit}

\subsection{Allgemeine Entwicklung der Beschäftigung bis 1914}

Die Arbeit im 17. und 18. Jahrhundert erfuhr im Zuge einer neuen Interpretation in der Literatur eine enorme Aufwertung. Arbeit wurde zum Kern menschlicher Selbstverwirklichung verklärt. Auch nach Marx gehört Arbeit ganz wesentlich zu einem sinnerfüllten Leben, wenngleich diese ihm zufolge im Kapitalismus nur als „entfremdet“ erfahren werden kann (Marx 1956: 512, Popitz 1953, Garaudy 1969 ). Heute ist Lohnarbeit in den Industrieländern die am weitesten verbreitete Form der Erwerbsarbeit. Dies war, wie bereits in einem vorangegangenen Artikel zum Normalarbeitsverhältnis gezeigt werden konnte, nicht immer der Fall (Pierenkemper/Kendzia 2010). Denn erst im Zuge der Industrialisierung begann „freie Lohnarbeit die herrschende Erwerbsform der Massen“ (Borchardt 1978: 39) zu werden.

Um 1800 kann man von einer täglichen Arbeitszeit von 10 bis 12 Stunden ausgehen, die dann tendenziell bis zu 14 bis 16 Stunden täglich und 80 bis 85 Stunden wöchentlich ausgedehnt wurde und damit die Grenze der physischen Leistungsfähigkeit des Menschen erreichte und gelegentlich auch überschritt. Erst zur Jahrhundertmitte setzte sich auch bei der Arbeitszeit allmählich eine Gegentendenz durch. Von 1850 bis 1870 blieb die tägliche Arbeitszeit relativ stabil und zeigte danach eine sinkende Tendenz, die mit einigen Neuerungen hinsichtlich der Aufteilung der Arbeitszeit verbunden war. Zunehmend wurde z. B. in Fabriken Schichtarbeit eingeführt und auch die ,englische“ Arbeitszeit mit kurzer Mittagspause fand weitgehend Verbreitung. Die sinkende Arbeitszeit hatte für die Unternehmer den Vorteil einer steigenden Arbeitsproduktivität und gab darüber hinaus den Arbeitern die Möglichkeit zu höher bezahlten Überstunden. Bis 1913 hatte sich dann der Zehnstundentag und in vielen Branchen die Sonntagsruhe weitgehend durchgesetzt (Pierenkemper 2009). 
Tabelle 1: Arbeitszeit in Deutschland von 1800 bis 1914

\begin{tabular}{|c|c|}
\hline Zeitraum & $\begin{array}{c}\text { Durchschnittliche tägli- } \\
\text { che Arbeitszeit in Stun- } \\
\text { den }\end{array}$ \\
\hline 1800 & $10-12$ \\
\hline 1820 & $11-14$ \\
\hline $1830-1860$ & $14-16$ \\
\hline $1861-1870$ & $12-14$ \\
\hline $1871-1880$ & 12 \\
\hline $1881-1890$ & 11 \\
\hline $1891-1895$ & $10,5-11$ \\
\hline $1896-1900$ & 10,5 \\
\hline $1901-1905$ & $10-10,5$ \\
\hline $1906-1910$ & $10-10,5$ \\
\hline $1911-1914$ & 10 \\
\hline
\end{tabular}

Quelle: Meinert 1958: 5-21.

Bezüglich der Löhne ist zwischen Akkord- beziehungsweise Stücklohn und dem Zeitlohn zu unterscheiden: Beim Akkord- oder Stücklohn richtet sich die Lohnhöhe nach dem Erfolg der Arbeit - also der tatsächlichen Arbeitsleistung. Für einen Berliner Großbetrieb in der Maschinenbauindustrie am Ende des 19. Jahrhunderts gibt Reichelt an, dass vor allem alle unproduktiven und ungelernten Hilfsarbeiter und nur wenige gelernte Arbeiter einen Zeitlohn in Form eines Stundenlohns erhielten. Dies war zu jener Zeit allgemein üblich. Dagegen bereitete die genaue Bestimmung der Akkordlöhne enorme Schwierigkeiten, weil hierfür die Messung der Leistung eines Arbeiters unerlässlich war. Die Berechnung des Zeitlohns, die Multiplikation der Arbeitsstunden mit dem Lohnsatz, war demzufolge einfacher, als der Akkordlohn. Wenn zum Beispiel eine Arbeit angefangen, aber noch nicht fertig gestellt worden konnte, erhielten die Arbeiter am Lohntag Vorschusszahlungen. Bei großen Aufträgen, die Wochen dauerten, bekamen die Arbeiter häufig nur ihre Stundenlohnsätze ausgezahlt, so dass in der Praxis teils Akkordlöhne, teils Zeitlöhne entrichtet wurden (Reichelt 1906: 21-47). 
Tabelle 2: Indexziffern der Lebenshaltungskosten, der Nominal- und Reallöhne in Deutschland 1888-1954 $(1913=100)$

\begin{tabular}{|c|c|c|c|c|c|}
\hline Jahr & $\begin{array}{l}\text { Lebens- } \\
\text { haltungs } \\
\text {-kosten }\end{array}$ & $\begin{array}{l}\text { Nominal } \\
\text { lohn }\end{array}$ & $\begin{array}{l}\text { Nominal- } \\
\text { lohn } \\
\text { (Kuczynski) }\end{array}$ & $\begin{array}{l}\text { Real- } \\
\text { lohn }\end{array}$ & $\begin{array}{l}\text { Real- } \\
\text { lohn } \\
\text { (Kuczynski) }\end{array}$ \\
\hline 1888 & 70 & 58,9 & 62 & 84 & 89 \\
\hline 1889 & 73,1 & 60,7 & 64 & 83 & 88 \\
\hline 1890 & 75,4 & 61,5 & 65 & 82 & 86 \\
\hline 1891 & 76,9 & 63,5 & 65 & 83 & 85 \\
\hline 1892 & 76,2 & 63,2 & 65 & 83 & 85 \\
\hline 1893 & 74,6 & 63,4 & 65 & 85 & 87 \\
\hline 1894 & 73,8 & 63,6 & 65 & 86 & 88 \\
\hline 1895 & 73,1 & 64 & 65 & 88 & 89 \\
\hline 1896 & 72,3 & 65,7 & 68 & 91 & 94 \\
\hline 1897 & 73,8 & 67,4 & 68 & 91 & 92 \\
\hline 1898 & 76,2 & 69,1 & 71 & 91 & 93 \\
\hline 1899 & 76,2 & 70,9 & 73 & 93 & 96 \\
\hline 1900 & 76,9 & 72,9 & 75 & 95 & 98 \\
\hline 1901 & 77,7 & 73,1 & 74 & 94 & 95 \\
\hline 1902 & 78,5 & 73,6 & 74 & 94 & 94 \\
\hline 1903 & 78,5 & 75,2 & 75 & 96 & 96 \\
\hline 1904 & 79,2 & 77 & 77 & 97 & 97 \\
\hline 1905 & 82,3 & 78,9 & 80 & 96 & 97 \\
\hline 1906 & 86,9 & 83,2 & 84 & 96 & 97 \\
\hline 1907 & 87,7 & 87,2 & 89 & 99 & 101 \\
\hline 1908 & 87,7 & 88 & 88 & 101 & 100 \\
\hline 1909 & 90 & 88,4 & 89 & 98 & 99 \\
\hline 1910 & 92,3 & 90,8 & 91 & 98 & 99 \\
\hline 1911 & 95,4 & 93,8 & 93 & 98 & 97 \\
\hline 1912 & 100 & 97 & 96 & 97 & 96 \\
\hline & & & & & \\
\hline
\end{tabular}

Quelle: Eigene Darstellung in Anlehnung an Grumbach/König 1957: 150, Kuczynski 1961: 173, 324, Kuczynski 1961: 170-171, 320 .

Die heutigen Industriegesellschaften sind durch unterschiedliche Ausprägungen des Lohnarbeitsverhältnisses gekennzeichnet. Dies hängt damit zusammen, dass nationale Gesellschaften unterschiedliche Entwicklungspfade beschritten. In Deutschland breitete sich die Lohnarbeit erst allmählich seit Anfang des 19. Jahrhunderts immer weiter aus. Die vollständige Genese der Erwerbsgesellschaft selbst, also die Durchsetzung von Marktbeziehungen als Mechanismus der Allokation gesellschaftlicher Arbeit, hat jedoch in Deutschland noch ein ganzes Jahrhundert gedauert (Pierenkemper 1984). Schon vor dem 19. Jahrhundert hatte es freie 
Lohnarbeit gegeben, doch stellte diese aus heutiger Sicht neben anderen (gebundenen) Formen der Arbeit eher ein Minderheitenphänomen dar. Erst im Zuge tiefgreifender Reformen innerhalb der Landwirtschaft und des Gewerbes wurden im 19. Jahrhundert die Voraussetzungen dafür geschaffen, dass Lohnarbeit zur dominierenden Form der Arbeitsverfassung wurde und die an Bodenbesitz und berufsständische Ordnung gebundenen Arbeitsformen immer mehr verdrängte. Dieser von Karl Polanyi als „Great Transformation“ bezeichnete Wandel führte zu einer grundlegenden Veränderung von Wirtschaft und Gesellschaft, welche den Boden für eine moderne Erwerbsgesellschaft bereitete (Pierenkemper 2006a: 64-65).

Die Durchsetzung der Lohnarbeit und die Entwicklung des Arbeitsmarktes in Deutschland im 19. Jahrhundert kann in seiner Wirkung nicht überschätzt werden (Kocka 1990). Während um 1800 die Lohnarbeit eher als ein Randphänomen in Erscheinung trat, war einhundert Jahre später die Mehrheit der arbeitenden Bevölkerung in ein Lohnarbeitsverhältnis eingebunden (Pierenkemper 2006a: 65-66). Lohnarbeit breitete sich also erst durch die Industrialisierung massenhaft aus - mit der Großindustrie als ihrer natürlichen Umgebung (Salais 1985, S. 342). Grundsätzlich lässt sich behaupten, dass sich die Arbeits- und Lebensbedingungen der Lohnarbeiter ab 1850 verbesserten (Borchardt 1978: 158). Hiermit hing auch die Hochkonjunktur bis 1873 zusammen, die allerdings durch den dann folgenden Gründerkrach und die Krise der Weltkonjunktur bis Februar 1879 deutlich gebremst wurde. Nach einer kurzen Erholungsphase bis 1882 dauerte die nächste, aber etwas schwächere Depression bis August 1886 an, ehe sich von 1895 bis 1913 wieder eine Hochkonjunktur einstellte (Wehler 1994: 42-43). Beim Übergang vom 19. ins 20. Jahrhundert hatte sich die Erwerbsgesellschaft bereits etablieren können. Lohnarbeitsverhältnisse und Arbeitsmärkte dienten als Allokationsmechanismen für die Organisation gesellschaftlicher Arbeit (Pierenkemper 2006a: 66). Generell lässt sich die Beschäftigung als Austauschvorgang der „Ware“ Arbeitskraft umschreiben (Pierenkemper 1984: 145). Im Folgenden wird nun ein Blick auf die Entwicklung der Beschäftigung in den verschiedenen Sektoren in Deutschland geworfen. 
Tabelle 3: Beschäftigte in den Sektoren der Wirtschaft (1800-1913)

\begin{tabular}{|c|c|c|c|}
\hline & \multicolumn{3}{|c|}{ in \% } \\
\hline & Landwirtschaft & Gewerbe & Dienstleistungen \\
\hline 1800 & 62 & 21 & 17 \\
\hline 1825 & 59 & 22 & 19 \\
\hline 1849 & 56 & 24 & 20 \\
\hline 1861 & 52 & 27 & 21 \\
\hline 1875 & 50 & 29 & 21 \\
\hline 1900 & 38 & 37 & 24 \\
\hline 1913 & 35 & 38 & 28 \\
\hline
\end{tabular}

Quelle: Fischer et al. 1982: 52-53, Hoffmann 1965: 205, Kocka 1990: 77.

Ebenso nach Wirtschaftsbereichen geordnet, ergibt sich nach Hoffmann für die Entwicklung der Beschäftigung in den deutschen Territorien, beziehungsweise nach der Reichsgründung von 1871 in Deutschland von 1846 bis 1914, nachstehende Tabelle. Folgt man den Angaben bei Hoffmann, dann übersteigt zu Beginn des 20. Jahrhunderts, laut Tabelle 4 im Jahr 1907, der sekundäre Sektor mit Blick auf die Anzahl der Beschäftigten erstmals die Anzahl der Beschäftigten im primären Sektor.

Tabelle 4: Die Beschäftigten nach Wirtschaftsbereichen 1846-1913 (in Tausend)

\begin{tabular}{|c|c|c|c|c|}
\hline Jahr & $\begin{array}{c}\text { Landwirtschaft, } \\
\text { Forsten, Fi- } \\
\text { scherei }\end{array}$ & $\begin{array}{c}\text { Industrie und } \\
\text { Handwerk }\end{array}$ & $\begin{array}{c}\text { Häusliche } \\
\text { Dienste }\end{array}$ & $\begin{array}{c}\text { Sonstige Dienst- } \\
\text { leistungen ohne } \\
\text { Verteidigung }\end{array}$ \\
\hline & 1 & 3 & 6 & 7 \\
\hline 1846 & - & 3305 & $(1400)$ & 398 \\
\hline 1849 & 8298 & 3396 & 1405 & 411 \\
\hline 1852 & 8293 & 3649 & 1361 & 432 \\
\hline 1855 & 8195 & 3709 & 1442 & 447 \\
\hline 1858 & 8235 & 3938 & 1403 & 457 \\
\hline 1861 & 8253 & 4187 & 1411 & 473 \\
\hline 1867 & 8333 & 4155 & 1422 & 508 \\
\hline 1871 & 8541 & 4762 & 1491 & 530 \\
\hline 1875 & 9230 & 5153 & $(1490)$ & 589 \\
\hline 1876 & $(9250)$ & 5327 & - & - \\
\hline
\end{tabular}




\begin{tabular}{|c|c|c|c|c|}
\hline 1877 & 9382 & 5346 & - & - \\
\hline 1878 & 9518 & 5300 & $(1484)$ & 628 \\
\hline 1879 & 9568 & 5468 & 1489 & 634 \\
\hline 1880 & 9565 & 5504 & 1492 & 632 \\
\hline 1881 & 9609 & 5492 & 1476 & 642 \\
\hline 1882 & 9665 & 5580 & 1487 & 645 \\
\hline 1883 & 9711 & 5691 & 1498 & 651 \\
\hline 1884 & 9698 & 5887 & 1508 & 652 \\
\hline 1885 & 9700 & 6005 & 1488 & 659 \\
\hline 1886 & 9740 & 6307 & 1486 & 672 \\
\hline 1887 & 9720 & 6489 & 1502 & 695 \\
\hline 1888 & 9645 & 6763 & 1525 & 723 \\
\hline 1889 & 9638 & 7123 & 1539 & 749 \\
\hline 1890 & 9565 & 7337 & 1532 & 778 \\
\hline 1891 & 9551 & 7343 & 1519 & 802 \\
\hline 1892 & 9543 & 7375 & 1512 & 823 \\
\hline 1893 & 9656 & 7311 & 1529 & 848 \\
\hline 1894 & 9765 & 7361 & 1541 & 870 \\
\hline 1895 & 9788 & 7524 & 1571 & 894 \\
\hline 1896 & 9778 & 7909 & 1567 & 915 \\
\hline 1897 & 9728 & 8211 & 1566 & 936 \\
\hline 1898 & 9720 & 8506 & 1544 & 959 \\
\hline 1899 & 9709 & 8741 & 1520 & 984 \\
\hline 1900 & 9754 & 8950 & 1508 & 1009 \\
\hline 1901 & 9825 & 8784 & 1502 & 1037 \\
\hline 1902 & 9947 & 8777 & 1503 & 1071 \\
\hline 1903 & 9987 & 9026 & 1509 & 1103 \\
\hline 1904 & 9999 & 9298 & 1518 & 1133 \\
\hline 1905 & 9926 & 9572 & 1541 & 1159 \\
\hline 1906 & 9888 & 9880 & 1568 & 1194 \\
\hline 1907 & 9897 & 10070 & 1581 & 1233 \\
\hline 1908 & 10096 & 9857 & 1583 & 1278 \\
\hline 1909 & 10350 & 9873 & 1571 & 1320 \\
\hline 1910 & 10542 & 10184 & 1570 & 1364 \\
\hline 1911 & 10627 & 10550 & 1569 & 1404 \\
\hline 1912 & 10663 & 10818 & 1567 & 1453 \\
\hline 1913 & 10701 & 10857 & 1542 & 1493 \\
\hline
\end{tabular}

Quelle: Eigene Darstellung in Anlehnung an Hoffmann 1965: 204-206. 


\subsection{Der Arbeitsvertrag}

Das 1896 entstandene Bürgerliche Gesetzbuch (BGB) betrachtet den Arbeitsvertrag als einen Schuldvertrag: es werden Dienste geschuldet. $§ 611$ Abs. 1 BGB besagt:

„Durch den Dienstvertrag wird derjenige, welcher Dienste zusagt, zur Leistung der versprochenen Dienste, der andere Teil zur Gewährung der vereinbarten Vergütung verpflichtet.“

Der Arbeitsvertrag begründet laut BGB also das Arbeitsverhältnis, deren Dienste weisungsgebunden und in abhängiger Arbeit geleistet werden. Während Arbeitgeber über Kapital verfügen, wie z.B. Produktionsmittel, Geld oder Waren und der Abschluss eines Arbeitsvertrages für sie vor allem eine Mehrung des eingesetzten Kapitals bedeutet, sind Lohnabhängige gezwungen, sich einem Arbeitsverhältnis zu unterwerfen. Vor diesem Hintergrund erscheint Mückenberger der Begriff „Lohnabhängiger“ insgesamt zutreffender als der Begriff „Arbeitnehmer“. Lohnarbeit stellt für den abhängig Beschäftigten eine existentielle Notwendigkeit dar. Trotz freiwilligen Abschlusses des Arbeitsvertrages sei das Arbeitsverhältnis daher als ein Herrschaftsverhältnis anzusehen, welches täglich neu bestätigt wird. Kern dieses Abhängigkeitsverhältnisses ist ein personenrechtliches Verhältnis, das auf dem Grundsatz beiderseitiger Treue basiert (Mückenberger 1985: 24-25). Auch andere Autoren betrachten das Arbeitsverhältnis als ein sozialisiertes Lohnverhältnis, welches durch die Willensunterwerfung von Personen einem Herrschaftsverband ähnelt. Nipperdey betont beim Arbeitsverhältnis vor allem die Treue- und Fürsorgepflichten des Arbeitsgebers (Becker 2005: 215-216, 298).

Erst die Industrialisierung führte zur Entstehung einer Lohnarbeitsgesellschaft. D.h., dass Lohnarbeit im Verlauf des 19. Jahrhunderts den Mittelpunkt der Gesellschaft, des Staates und der meisten Biographien bildete. Der Lohn determinierte fortan in Form des Einkommens die Konsummuster und Lebensweise der Beschäftigten. Er hatte zudem Auswirkungen auf Produktion, Arbeitsdisziplin sowie den Rechtsstatus im Arbeitsvertrag. Arbeit wurde zur Lohnarbeit, zur Ware, und die Marktabhängigkeit der Arbeitskraft trat an die Stelle traditioneller Arbeits- und Lebensformen in Landwirtschaft, Handwerk und Hauswirtschaft. Hierdurch kam es auch zu einer Trennung von Arbeit und Leben und eine klare Unterscheidung von Arbeit und Freizeit wurde möglich. Der Arbeitsplatz konnte als eine abgrenzbare und dauerhafte Tätigkeit interpretiert werden (Pierenkemper 2006a: 60-61). Für die Arbeiter hieß dies, dass sich allmählich eine Normalform des Arbeitsverhältnisses durchsetzte. Diese Form der Beschäftigung bildete das so genannte Normalarbeitsverhältnis. 
Die Durchsetzung von Lohnarbeitsverhältnissen erfolgte nach Castel in drei Stufen. Zunächst bildete sich während der Frühindustrialisierung der Typ eines modernen Proletariers heraus. Anschließend, auf der zweiten Stufe der Hochindustrialisierung, nahm die soziale Stellung des Industriearbeiters zu. Der Lohnarbeiter wurde mit Rechten ausgestattet, die ihm den Zugang zu bestimmten Leistungen (bei Krankheit, Unfällen, Alter, etc.) und erweiterte Teilhabe am sozialen Leben (Wohnung, Bildung, Konsum, etc.) ermöglichten. Auf der letzten Stufe angekommen, umfasste die Lohnabhängigkeit den Großteil aller Erwerbstätigen. In der Folge entstanden neue Beschäftigungsgruppen (Castel 2000: 193-285). Aufgrund immer größerer Betriebe und einer Intensivierung der Arbeitsteilung nahm auch die Zahl der Angestellten rapide zu. Diese bildeten einen neuen Mittelstand, der sich zwischen Arbeitern und dem Bürgertum etablierte (Schmuhl 2003: 5).

\subsection{Das deutsche Berufsbeamtentum}

Das deutsche Berufsbeamtentum entstand im frühen 19. Jahrhundert als Teil eines neuen Rechtsstaates, der zugleich die Herausbildung einer bürgerlichen Gesellschaft und eines kapitalistischen Systems ermöglichte. Obwohl sich zu jener Zeit das Prinzip des Wettbewerbs durchsetzte, wurden die Beamten, so Wilhelm Heinrich Riehl zur Mitte des 19. Jahrhunderts, in einen „künstlichen Stand“ versetzt und konnten sich somit der Konkurrenz entziehen. Ziel dieses eigenen Standes war es, ein Organ für die Regierung zu schaffen, welches unabhängig von sozialen und wirtschaftlichen Strömungen die Aufrechterhaltung staatlicher Tätigkeiten garantierte. Die Gewährung einer lebenslangen Anstellung für die zu erbringenden Dienstleistungen des Berufsbeamtentums auf der einen Seite, verlangte auf der anderen Seite unbedingten Gehorsam gegenüber den Vorgesetzten (Wunder 1986: 66).

Um das Berufsbeamtentum vor der sozialdemokratischen Arbeiterbewegung im 19. Jahrhundert zu schützen, führte die Regierung das „Zuckerbrot“ der Verbeamtung beziehungsweise Unkündbarkeit ein. Als Gegenleistung, quasi als „Peitsche“, erfolgte dann eine strengere Disziplinierung durch das Koalitionsverbot. Die Beamtenschaft nahm aufgrund ihrer ,institutionellen Nähe zur Regierung“" weiterhin eine privilegierte Stellung innerhalb der Gesellschaft ein. Ihr hohes Ansehen offenbarte sich auch in dem Bestreben der entstehenden Angestelltenschaft in der Industrie, sich als Privatbeamte zu bezeichnen - so jedenfalls deren Bezeichnung bis 1911 (Wunder 1986: 107-108).

Bereits im preußischen Allgemeinen Landrecht von 1794, das noch das Ämterprivileg des Adels bestätigte, war den Staatsdienern eine besondere Stellung eingeräumt worden. Juris- 
tisch bedeutete dies, dass das Arbeitsverhältnis der Beamten aus dem privatrechtlichen Dienst- und Arbeitsrecht gelöst und zu einem öffentlich-rechtlichen Dienstverhältnis erklärt wurde. Die erste rechtliche Fixierung des Beamtenrechts fand 1808 in Bayern statt: Die bayrische Hauptlandespragmatik regelte die Dienstverhältnisse der Staatsdiener. Es folgten weitere deutsche Staaten, 1850 schließlich auch Preußen. Hierdurch wurde die Unkündbarkeit der Beamten in die jeweiligen Verfassungen aufgenommen. Eine Entlassung war anschließend nur bei grober Verletzung der Dienstpflichten möglich, die in einem quasi-gerichtlichen Verfahren festgestellt werden musste (Wunder 1986: 28-30).

Die fachliche Qualifikation stellte allerdings nur ein sekundäres Selektionskriterium bei der Rekrutierung dar, denn den damaligen Vorstellungen entsprechend, waren die Herrschaftspositionen in erster Linie einer geburtsständischen Elite vorbehalten - die Rekrutierung fand also nach sozialen Erwägungen statt. Wenn die fachliche Qualifikation der Beamten nur eine sekundäre Eigenschaft darstellte, dann so Wunder, war die entscheidende Eigenschaft die Treue des Beamten, also das Treueverhältnis zum Staatsoberhaupt. Dieses bildete den Kern des Dienstverhältnisses zwischen dem jeweiligen Regenten und dem Beamten und änderte sich erst durch die Demokratisierung der Verwaltung zur Weimarer Zeit (Wunder 1979: 1221). Der seit 1881 amtierende preußische Innenminister Puttkamer verlangte 1883 sogar ,die absolute, willenlose Unterordnung des Beamtenstandes unter den Willen des allmächtigen Ministers“(Rejewski 1973: 90).

Das Reichsbeamtengesetz (RBG) vom 31. März 1873 galt - mit nur wenigen Änderungen durch die Bekanntmachung vom 18. Mai 1907 - für die Zeit des Kaiserreichs und die Weimarer Republik. Letztere war für die Traditionsbildung des Berufsbeamtentums von großer Bedeutung. Das RGB regelte die Dienstverhältnisse der Staatsdiener. Das Anhaltische Beamtengesetz (Zivilstaatsdienergesetz für die Herzogtümer Anhalt-Dessau und AnhaltKöthen vom 10. April 1850) definierte die Staatsdiener als diejenigen Personen, denen seitens der Staatsgewalt ein öffentliches Amt für die Zwecke des Staates anvertraut wurde. § 2 RBG regelte die lebenslange Anstellung des Reichsbeamten (Krause 2008: 9-13).

Das Berufsbeamtentum ist nach Krause weder auf die Gründung des modernen Staates noch auf die Industrialisierung zurückzuführen. Eher erscheint die Grundherrschaft, die „Herrschaft über Land und Leute“ als Strukturprinzip der Ausübung hoheitlicher Kompetenzen zu gelten: Einerseits bestanden Schutz- und Fürsorgepflichten des Staates gegenüber dem Beamten, andererseits war der Beamte zu Treue und Dienstbereitschaft angehalten (Krause 2008: 68-70, 86). 
Das Gesinderechtsverhältnis hingegen war ein eher privatautonomes vertragliches Verhältnis. Mit dem Eintritt in das Gesindeverhältnis waren automatisch deutliche Einschränkungen in der persönlichen Freiheit und des Persönlichkeitsrechts verbunden. Etwa durch die Pflicht, die Befehle der Herrschaft mit Ehrerbietung und Bescheidenheit anzunehmen. Damit weist das privatrechtliche Gesindeverhältnis eine deutliche Orientierung an der Grundherrschaft auf, so dass es mit einem Berufsbeamtenverhältnis zumindest nicht im Widerspruch steht. Gleichwohl war das Gesindeverhältnis nicht zur Hervorbringung des Berufsbeamtentums fähig, da es immer an der Hausgemeinschaft zwischen Herren und Bediensteten festhielt. Das Element der Außerhäuslichkeit im Beamtenrecht war nie Teil des Gesinderechts (Krause 2008: 142-144).

Das Rechtsverhältnis des Berufsbeamtentums löste die Lehnsherrschaft von Personen ab, die mit hoheitlichen Aufgaben betraut wurden. Die immanente Treuepflicht der Lehnsherrschaft wurde Teil des Berufsbeamtentums, das nach dem Ende der Stauferherrschaft in gewissen Grundzügen erkennbar ist. Das Berufsbeamtenverhältnis ist damit älter als viele erst in der Neuzeit entstandene Staaten. Dies führt Krause darauf zurück, dass das Berufsbeamtentum für die Bildung eines Staates erforderlich war (Krause 2008: 315).

Merz gibt an, dass die Lebenszeitdauer der Beamten nicht im Entstehungszusammenhang des Berufsbeamtentums zu sehen ist. Vielmehr sollte die materielle Versorgung der Beamten sichergestellt und eine enge und konkurrenzlose Bindung an den Staat gewährleistet werden, so dass der Beamte als Sachverwalter allgemeiner Interessen das Wohl des Staates fördern konnte (Merz 1985: 2-3). Dem Ansinnen des Staatsrechtslehrers Nikolaus Thaddäus Gönner entsprechend sollte der Staatsbeamte als eine Maschine zur Ausübung der Staatsverfassung dienen (Stirken 1984: 157-158).

Trotz deutlicher Abgrenzung des Themas von Angehörigen der geistlichen Sphäre - auch Schmoller argumentiert, dass Kirchenämter vom weltlichen Amt grundsätzlich als „,wesensverschieden“ aufzufassen sind (Schmoller 1894: 36) - stellt Krause klar, dass „Adel und Klerus Kehrseiten ein- und derselben gesellschaftlichen Schicht waren“ (Krause 2008: 63). Das Herrschaftssystem der Kirche weist keine allzu großen strukturellen Unterscheidungsmerkmale zum Lehnswesen auf. Im Gegenteil: Während im Lehnswesen der König oder Kaiser die oberste Stellung innerhalb der Hierarchie einnahm, nimmt im kirchlichen Herrschaftssystem der Papst die eben erwähnte Position ein. Die eingeräumten Ämter wurden dem einzelnen Amtsträger in der Regel auf unbestimmte Zeit übertragen. Lediglich schwerwiegende Vergehen führten nach einem festgelegten Verfahren zur Amtsenthebung (Boehmer 1789: 64-65). 
Für die weitere Untersuchung von großem Interesse bleibt die nähere Ausgestaltung des Berufsbeamtenverhältnisses. Den Beamten gewährte der Staat eine dauerhafte und nahezu unkündbare Anstellung, wofür er im Gegenzug eine geradezu bedingungslose Gefolgschaft beziehungsweise Loyalität einforderte. Abschließend lässt sich festhalten, dass Berufsbeamtentum und Kirchenämter gewisse strukturelle Analogien aufweisen. 


\title{
3 Entstehung der Sozialen Frage im 19. Jahrhundert
}

\subsection{Triebkräfte der Sozialen Frage}

Die Soziale Frage „wurde von vielen Zeitgenossen als das Kernproblem des ausgehenden 19. Jahrhunderts empfunden“ (Schmuhl 2003: 6). Für einen immer größeren Teil der Bevölkerung bildete das Arbeitseinkommen die einzige Quelle des Lebensunterhalts. Unregelmäßige Arbeit führte demzufolge immer häufiger auch zu Einschränkungen in der Lebensführung. Die sich hieraus ergebenden sozialen Schieflagen eines breiten Teils der Bevölkerung wurden im 19. Jahrhundert als die „Soziale Frage“ thematisiert (Alber 1982: 32), wie beispielsweise durch Gustav Scholler in seinem gleichnamigen Werk:

\begin{abstract}
„Erst die letzten hundert Jahre aber haben die Beseitigung der Hörigkeit in Europa [...] in der Form der Durchführung großer Emanzipationsgesetze, welche von den staatlichen Gewalten angeordnet, im Laufe einiger Jahre oder Generationen die große rechtliche und wirtschaftliche Umbildung vollzogen. Sie brachte den handarbeitenden Klassen die persönliche Freiheit, die Gleichstellung in bezug auf Ehe, Eigentum, Freizügigkeit, Berufswahl, Vertragsfreiheit mit den übrigen Klassen, sie schufen formell und rechtlich, definitiv und allgemein einen freien Arbeiterstand. [...] Auch soweit bisher schon freie Arbeiter existiert hatten, standen sie doch vielfach durch die patriarchalische oder koporative Verfassung, durch Naturallöhne mangelnde Gewerbefreiheit und Freizügigkeit, durch erbliche Sitte unter einer analogen Sicherung ihres Lebens wie unter einer gewissen Leitung von oben. Auch sie sollten nun ganz auf sich stehen, durch freie Verträge, durch Geldlohn sich eine gesicherte, gute Existenz schaffen“ (Schmoller 1918: 193-194).
\end{abstract}

Ingesamt war der Übergang der Gesellschaft in ein neues Zeitalter durch mehrere vorherrschende Trends gekennzeichnet. Schmuhl identifiziert insgesamt sechs so genannte Basisprozesse: Bevölkerungsexplosion, Urbanisierung, Wanderungsbewegungen, Industrialisierung, Klassenbildung und Entstehung des Sozialstaats (Schmuhl 2003: 8).

Die Bevölkerung des Deutschen Reichs wuchs von 41 Mio. im Jahr 1871 bis auf 67,8 Mio. im Jahr 1914 an (Schmuhl 2003: 1). Ursächlich für das rapide Bevölkerungswachstum war der so genannte demographische Übergang. Dieser wird definiert als die Übergangsphase von einer traditionellen Bevölkerungsweise mit hoher Fertilität und hoher Mortalität zu einer modernen Bevölkerungsweise mit niedriger Fertilität und Mortalität. Im Übergang sinkt zunächst die Mortalität, wohingegen die Fertilität erst mit einiger Verzögerung zu sinken beginnt, so dass die Bevölkerung in dieser Phase einen starken Zuwachs erfährt (Marschalck 1984: 41-71).

Für die Unterschichten in der vorindustriellen Zeit bedeutete Armut eine nahezu alltägliche Erfahrung. Von Hippel zählt zu diesem Kreis Tagelöhner, Handwerker, Heimarbeiter und selbst zahlreiche Angehörige des Militärs. Weit über die Mitte des 19. Jahrhunderts hinweg 
war insbesondere diese Gruppe gezwungen, 65 bis 70\% ihres Einkommens für Nahrungsmittel aufzuwenden - in den vorangegangenen Dekaden dürfte dieser Anteil um 5 bis 10\% höher gewesen sein. Dies hatte zur Folge, dass Missernten unmittelbar und mit aller Schärfe zu mehr Armut führten (von Hippel 1995: 3-13). Die Löhne stagnierten über Jahrhunderte hinweg auf einem äußerst niedrigen Niveau und auch die Arbeitsmöglichkeiten selbst waren auBerordentlich rar. Ganz unverhüllt kann daher behauptet werden, dass die europäische Gesellschaft im vorindustriellen Zeitalter arm gewesen ist (Pierenkemper 2005: 15-16).

Wegen unterschiedlicher regionaler Entwicklung von Bevölkerung und Industrie mit entsprechenden Arbeitsplätzen entstanden deutliche Ungleichgewichte, die in Außen- und Binnenwanderungen zum Ausgleich strebten. Die Binnenwanderungsbewegung in der Hochindustrialisierung war die größte Massenwanderung der deutschen Geschichte. Fast jeder zweite Deutsche hat in diesem Zeitraum an der Binnenwanderungsbewegung teilgenommen. Hinzu kam die Außenwanderung, die sich zu Beginn des 19. Jahrhunderts vor allem aus dem südwestdeutschen Raum speiste und später in mehreren großen Auswanderungswellen auf West-, Mittel- und Ostdeutschland übergriff. Von geringerer Bedeutung war hingegen die Einwanderung nach Deutschland, die sich in der Frühphase der Industrialisierung vorwiegend auf englische Fachkräfte und später auf landwirtschaftliche Arbeitskräfte in den preußischen Nordostprovinzen beschränkte. Insgesamt lässt sich jedoch festhalten, dass der Umfang des Arbeitskräftepotentials durch das Bevölkerungswachstum im 19. Jahrhundert stark zunahm und wegen der industriellen Expansion zugleich die über den Arbeitsmarkt vermittelte Beschäftigung wuchs (Pierenkemper 1984).

Bedingt durch die Auflösung des Abhängigkeitsverhältnisses zwischen Grundherrn und Untertan, war der Grundherr auch nicht mehr dazu verpflichtet, seinen Untertanen im Falle der Not zu versorgen. „Erst die doppelte Freiheit des Lohnabhängigen - frei von persönlichen Bindungen und frei von eigenen Produktionsmitteln - macht ihm das Überleben, Essen, Wohnung, Liebe, Fortpflanzung, biologische und soziale Gesundheit zum persönlichen Problem, das er „privat“ zu lösen hat“" (Mückenberger 1985: 17-18).

Nunmehr waren die neuen und immer mehr werdenden Lohn- beziehungsweise Industriearbeiter auf sich alleine gestellt. An Sicherungseinrichtungen gegen die Risiken des Lebens wie Arbeitseinkommensverlust durch Arbeitslosigkeit, Krankheit, Unfall, vorzeitige Erwerbsunfähigkeit, Alter und Tod herrschte Mangel. Es gab bereits eine Menge an kleinen, berufsund branchengebundenen lokalen Unterstützungskassen zur Unterstützung von Kranken, Arbeitsunfähigen, Witwen und Waisen, jedoch hatten lediglich Arbeiter mit relativ stabilen und hohen Wochenlöhnen Zugang zu derartigen Kassen. Das Gros der Tagelöhner konnte die Bei- 
träge nicht aufbringen. Letztgenannte Gruppe konnte, wenn überhaupt, nur auf die Armenfürsorge der Gemeinden hoffen. Es bestanden zahlreiche soziale Defizite: Vor allem fehlte ein Schutz der Arbeitskraft vor körperlicher und psychischer Beeinträchtigung am Arbeitsplatz. Außerdem mangelte es an einem vorbeugenden Schutz vor zu langen Arbeitszeiten. Dennoch blieb den meisten nichts anderes übrig, als ihre Arbeitskraft auf dem neu entstandenen Arbeitsmarkt anzubieten (Lampert 1997: 10-11).

\subsection{Frühe Formen der sozialen Sicherung}

Frühe Formen der sozialen Sicherung, an die die bismarckschen Reformen anknüpften, waren z. B. die genossenschaftliche Unterstützung der Zünfte und Gilden, während der Feudalherrschaft das Schutz-Treue-Verhältnis zwischen Feudalherren und Untertanen sowie die kommunale Armenfürsorge seit dem 16. Jahrhundert. Im Jahr 1794 schrieb das Preußische Allgemeine Landrecht (ALR) vor, dass der Arbeitgeber sein Dienstpersonal im Krankheitsfall zu unterstützen habe. Einzelne Gewerbeordnungen in Europa beinhalteten Haftverpflichtungen für die Arbeitgeber bei Unfällen ihrer Arbeiter. Den eigentlichen Anfang der Ausübung sozialer Sicherung durch den Staat machte die Armenpflege zur Reformationszeit im 16. Jahrhundert. Bis zu dieser Zeit übernahmen Kirchengemeinden und Klöster derartige Leistungen. Erste Akzente im Bereich der staatlichen Daseinsfürsorge setzte England 1573 mit der Einführung einer Armensteuer auf Grundeigentum. Diese wurde im Nachhinein zu einer kommunalen Armenpflege unter staatlicher Leitung weiterentwickelt. Im weiteren Verlauf schufen immer mehr europäische Länder gesetzliche Armenordnungen (Alber 1982: 19-26). Wer zu früheren Zeiten auf die Unterstützung der Armenpflege angewiesen war, verlor zugleich seine Bürgerrechte. Nach dem ALR von 1794, wonach das Prinzip des Heimatrechts bei der Armenfürsorge griff, wurde dieses 1842 durch das Prinzip des Unterstützungswohnsitzes abgelöst. Hierdurch war die Gemeinde für die Unterstützung zuständig, in der die Person zuvor gewohnt hatte (Volkmann 1968: 48).

Regelrechte Berühmtheit erlangte das so genannte Elberfelder Modell. Elberfeld wuchs bis zur Mitte des 19. Jahrhunderts zu einer großen Industriestadt mit bereits 50.000 Industriearbeitern heran. Im Jahr 1852 wurde in der Stadt die kommunale Hausarmenpflege beschlossen. Hierfür teilte man die Stadt in zunächst 150 Quartiere auf, denen (ehrenamtliche) Armenpfleger, die wiederum von Seiten der Kirchen vorgeschlagen wurden, vorstanden. Die einzelnen Quartiere waren in insgesamt 18 Bezirke in Elberfeld eingeteilt, die einen Armenvorsteher hatten. Alle vierzehn Tage (genauso lange galt die Feststellung der Bedürftigkeit) 
traten die Armenpfleger in einer Bezirksversammlung zusammen und berieten im Einzelfall über potentielle Hilfen. Unterstützungen wurden im konkreten Notfall lediglich ausnahmsweise und äußerst gering gewährt. Zu den wichtigsten Aufgaben des Armenpflegers gehörte die Vermittlung des Betroffenen in Arbeit, weshalb sich mit der Zeit die Zahl der Unterstützungsanträge verringerte und das Modell in ganz Europa imitiert wurde. Das Modell wurde insbesondere aufgrund der inhärenten Effizienz bevorzugt, schließlich kosteten die ehrenamtlichen Pfleger nichts und die Ausübung ihrer Arbeit war von einer großen Sparsamkeit im Umgang mit Unterstützungsleistungen gekennzeichnet (Wendt 2008: 329-330).

\subsection{Unternehmerische und andere nicht-staatliche Initiativen}

Noch vor dem Beginn der staatlichen Sozialpolitik entwickelten sich in der Mitte des 19. Jahrhunderts unternehmerische Initiativen zur Verbesserung der Situation der Arbeiter. So schreibt Herkner im Jahr 1922, dass wohl keine sozialen Reformen ohne die Beteiligung der Arbeitgeber bewerkstelligt werden könnten (Herkner 1922: 443). Die so genannte Gründerzeit kann als eine „beispiellose Aufschwungperiode der 70er Jahre” (Reichelt 1906: 5) des 19. Jahrhunderts in Deutschland betrachtet werden.

Sozialpolitische Aktivitäten von Unternehmern finden sich in zahlreichen Biographien wie z. B. in der von Ernst Abbe, Robert Bosch, Heinrich Freese, Franz Haniel, Freiherr von Heyl, Alfred Krupp und vielen anderen. Unternehmer kümmerten sich demnach schon früh um die Belange ihrer Arbeiterschaft. Es entstanden zahlreiche unkoordinierte Ansätze sozialen Handelns, die gemeinsam das Ziel verfolgten, Arbeitskräfte für den Produktionsprozess zu halten (Bildung einer Stammbelegschaft) und durch Zusicherung von Loyalität die Arbeitsproduktivität zu erhöhen. Im Folgenden werden einige unternehmerische Initiativen genauer beleuchtet.

Alfred Krupp beispielsweise sah sich in der Rolle eines Arbeiterpatriarchen, dem auf der einen Seite das Recht zustand, Herr im eigenen Hause zu sein, der auf der anderen Seite aber auch die Pflicht hatte, für alle seine Angehörigen wie ein Vater zu sorgen. Bereits ab 1856 wurden Wohnhäuser für Arbeiter gebaut, um dem Wohnungsmangel der damaligen Zeit entgegenzuwirken. Es folgten ganze Wohnanlagen für seine Arbeiter. Im Jahr 1869 wurde eine Dampfmühle, 1871 eine Kaffeebrennerei, ein Jahr später eine Selterswasserfabrik und ein Gasthaus, 1874 eine Möbelhandlung, 1875 eine Metzgerei mit Fleischverkauf und 1871 das erste Epidemienhaus errichtet. Seit den 50er Jahren existierten Kranken-, Sterbe-, Pensionsund Unterstützungskassen, die durch Beiträge der Fabrik und der Arbeiter finanziert wurden. 
Im Gegenzug erwartete Krupp von seinen Arbeitern eine gewisse Treue gegenüber ihrem Arbeitgeber. Im Jahr 1875 entstand eine Industrieschule. Die Unterstützungskassen für Krankheits- und Todesfälle wuchsen mit der Zeit immer weiter an: Wurden 1856 insgesamt 1.750 Reichsmark ausgezahlt, waren es 1874 bereits 267.000 Reichsmark, die sich paritätisch aus den Zahlungen der Fabrik und der Arbeiter zusammensetzten und in insgesamt 22.474 Fällen in Anspruch genommen wurden (Tesch 1923: 72-89). Alfred Krupp hatte demnach bereits frühzeitig und freiwillig eine Vorstufe zu einer Betriebskrankenkasse geschaffen (Boeckh et al. 2004: 52).

Ähnlich verhielt es sich bei Ernst Abbe, der den Gewinn als das Ergebnis der organisierten Zusammenarbeit aller daran Beteiligten innerhalb der Zeissschen Werkstätten ansah. Abbe schuf mit der Carl-Zeiss-Stiftung eine neue Form der industriellen Arbeitsorganisation, und ermöglichte auch eine rechtliche Sicherstellung der arbeitenden und pensionierten Geschäftsangehörigen. Des Weiteren führte er schon vor dem Jahr 1900 den Achtstundentag ein, gewährte allen Arbeitern und Angestellten einen regelmäßigen Urlaub, garantierte einen Pensionsanspruch und zahlte Abgangsentschädigungen aus. Jegliche Mitbestimmung der Arbeiter bei dem Stiftungsunternehmen lehnte Abbe strikt ab, ebenso einen festen Anspruch der Arbeiter auf den Reingewinn. Die Lohn- und Gehaltsnachzahlungen (Prämienzahlungen) nach Abschluss eines jeden Geschäftsjahres waren für ihn ein adäquates Mittel um Schwankungen der wirtschaftlichen Konjunktur auszugleichen (Volkmann 1966: 34-36).

Robert Bosch führte im Jahr 1894 die neunstündige Arbeitszeit ein und stellte auch den Achtstundentag in Aussicht. Heinrich Freese, ein Berliner Jalousiefabrikant, führte 1892 als erster größerer Betrieb in Deutschland den Achtstundentag ein. Nach Abbe und Freese führte Bosch schließlich 1906 auf freiwilliger Basis den Achtstundentag ein (Heuss 1946: 167).

Nach Mommsen resultiert der Sozialstaat aus Industriezeitalter und Kapitalismus, der ohne einen gewaltigen technischen Fortschritt und der Emporhebung der Arbeiterbewegung in der Form niemals möglich geworden wäre (Mommsen 1981: 78). Je mehr sich Deutschland von einem Agrar- zu einem Industriestaat wandelte, desto stärker trat die Stellung der Lohnarbeiter in den Mittelpunkt der politischen Betrachtung (Manes 1928: 2).

Als einer der führenden Wortführer der deutschen Arbeiterbewegung gilt Ferdinand Lassalle. Er gehörte $1863 \mathrm{zu}$ den Mitbegründern des Allgemeinen Deutschen Arbeitervereins (ADAV). Der Verein forderte vor allem die Einführung des allgemeinen, gleichen und freien Wahlrechts (Euchner 2005: 141-145). Daneben gab es noch eine Reihe anderer Vertreter, die sich für die Arbeiterbewegung stark machten, wie beispielsweise Friedrich Albert Lange oder Johann Jacoby (Nipperdey 1995: 356). Der Verein für Socialpolitik, welcher 1872 von Gustav 
Schmoller und Adolph Wagner gegründet wurde, hatte das Ziel, in Deutschland Sozialreformen über Kongresse, Veranstaltungen und Veröffentlichungen zu forcieren. Im Jahr 1874 wurde beispielsweise das Werk Schmollers „Die soziale Frage und der preußische Staat“" veröffentlicht, in dem die unzureichende Behandlung der sozialen Lage der Arbeiter durch die Politik kritisiert wurde. Im Jahr 1854 waren in Preußen 246.000 Mitglieder in 2.622 Unterstützungskassen organisiert. Sechs Jahre später gab es bereits 3.644 Kassen mit 427.190 Mitgliedern. Damit waren erst $45 \%$ der preußischen Fabrikarbeiter erfasst, und qualitativ nur notdürftig abgesichert. Es bildeten sich Dachverbände mit drei Prinzipien: Beitragsfinanzierung sozialer Versicherungsleistungen inklusive eines Rechtsanspruches, solidarischer Ausgleich bei sozialen Risiken und Selbstverwaltung der Organisation durch die Versicherten. Hierdurch wurden im Grunde bereits wesentliche strukturelle Elemente der späteren Sozialversicherungen bestimmt (Boeckh et al. 2004: 56-61). Im Jahr 1880 gründete sich der „Deutsche Verein für Armenpflege und Wohltätigkeit“, der es sich zur Aufgabe machte, Fragen der Armenpflege und der Wohltätigkeit sowie ihre praktische Anwendung zu diskutieren und auf die Gesetzgebung einzuwirken (Wendt 2008: 195-196). 
Abbildung 1: Armen- und Sozialpolitik im Überblick
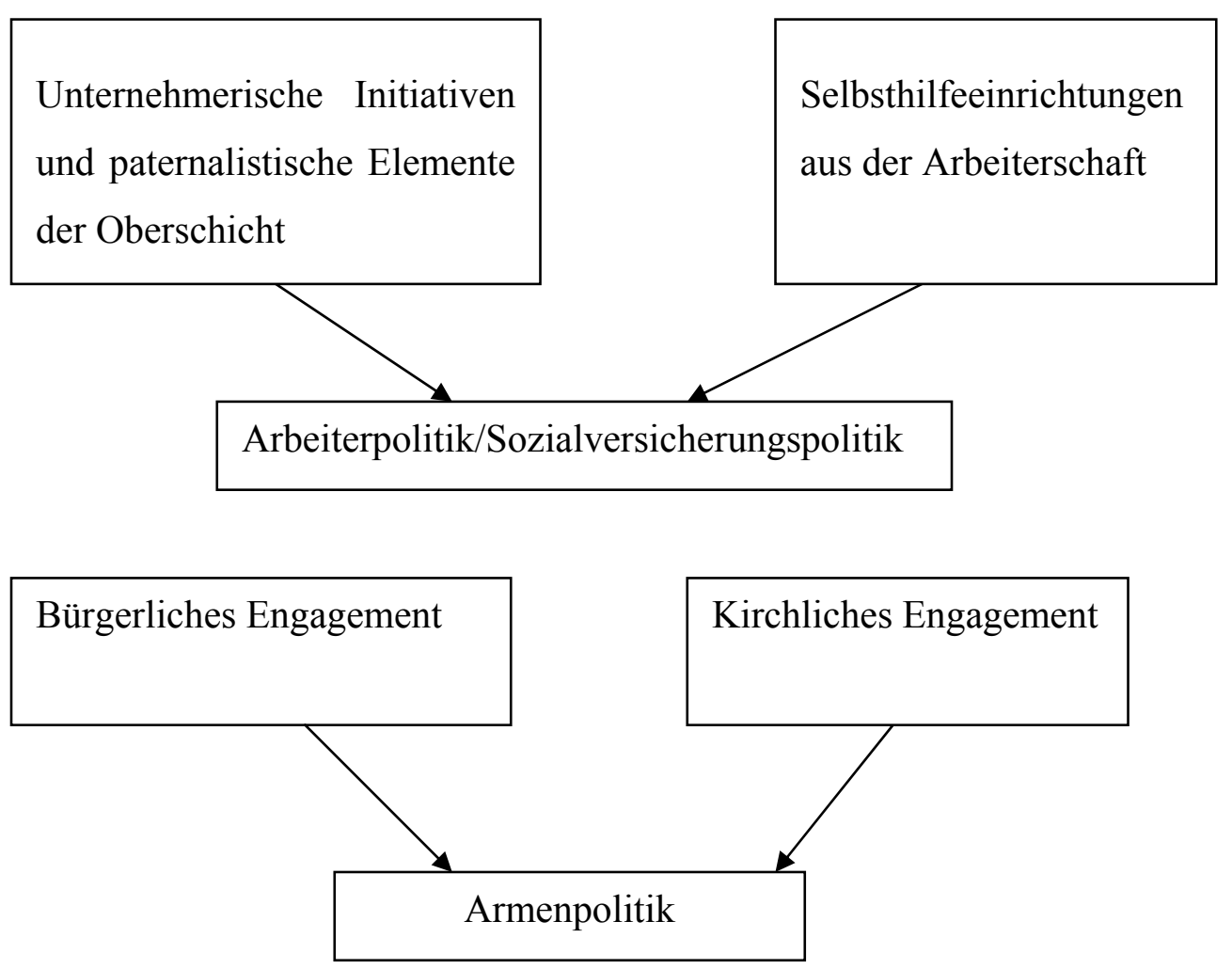

Quelle: Eigene Darstellung in Anlehnung an die Angaben bei Boeckh et al. 2004: 58. 


\title{
4 Institutionalisierung der Sozialversicherungssysteme durch Bismarck
}

Durch die Institutionalisierung der Sozialversicherungssysteme vollzog der Staat im Umgang mit der Armenpflege eine Kehrtwende. Hielt man Armut bisher für individuell verschuldet, so erkannte der Staat Notlagen nun als gesellschaftliches Problem an. Als Entstehungsphase der Sozialversicherung im westlichen Teil Europas lassen sich generell die 30 Jahre von 1885 bis 1915 anführen. Mit dem Verlesen der kaiserlichen Botschaft am 17. November 1881 anlässlich der Einführung der fünften Sitzungsperiode des Reichstages begann das Zeitalter staatlicher Sozialpolitik. Das deutsche Reich übernahm mit dem Aufbau einer verpflichtenden staatlichen Sozialversicherung eine Pionierrolle innerhalb Europas und damit auch in der Welt (Alber 1982: 19-29). Manes definiert eine Sozialversicherung wie folgt:

\begin{abstract}
„Vom wirtschaftswissenschaftlichen Standpunkt aus sind als Sozialversicherung im weitesten Sinne des Wortes zu bezeichnen alle auf Gegenseitigkeit beruhenden wirtschaftlichen Veranstaltungen, welche der Deckung zufälligen schätzbaren Vermögensbedarf notleidender Bevölkerungsschichten dienen. Die Bedarfsfälle entstehen durch Beeinträchtigung oder Aufhören der Arbeitsfähigkeit, oder durch ihre Unverwendbarkeit. Tod, Alter, Invalidität, Unfall, Krankheit, Schwangerschaft, Arbeitslosigkeit kommen hier vornehmlich als Ursachen in Betracht. Die Art der Begriffsbestimmung zeigt bereits, dass die Sozialversicherung als eine echte Versicherung aufzufassen ist, als eine Versicherung ebenso wie die zahlreichen Arten der Privatversicherung. Während aber für die Organisation der Privatversicherung privatwirtschaftliche Gesichtspunkte den Ausschlag geben, sind es hier solche der Sozialpolitik; daher die Bezeichnung Sozialversicherung, während die früher übliche Benennung Arbeiterversicherung sich überlebt hat, da nicht nur Arbeiter von der Versicherung erfasst werden, sondern auch Angestellte, z.T. auch selbständige Kleinunternehmer" (Manes 1928: 5-6).
\end{abstract}

Im engeren Sinn, so Manes weiter, ist als Sozialversicherung eine auf staatlichen Zwang basierende Versicherung aufzufassen. Des Weiteren dient der Begriff der „Sozialversicherung“ im Grunde genommen als ein Sammelbegriff für ein System verschiedener Versicherungsarten. Wie jede Versicherung bildet sie einen Teil der wirtschaftlichen Vorsorge. Als Einrichtung der öffentlichen Fürsorge wird sie dem öffentlichen Recht zugeschrieben (Manes 1928: 6-10). Ferner wird die Sozialversicherung durch zwei bestimmende Elemente geprägt: einmal durch die soziale Komponente, die sich in der Berechnung allgemeiner Beiträge nicht am Charakter des Versicherten (Alter, Gesundheitszustand, etc.) bemisst. Das zweite Element ist die Versicherung selbst. Eine Versicherung mit generellen Prämien ist nur dann möglich, wenn eine Versicherungspflicht besteht. Dafür erhält der Sozialversicherte einen Rechtsanspruch auf die entsprechende Versicherungsleistung, sofern diese benötigt wird (Molitor 1987: 11-12). Skeptisch stand Theodor Lohmann, der führende Kopf neben Bismarck beim Aufbau der deutschen Sozialversicherung, dem sozialpolitischen Nutzen der sozialen Versicherungsgesetzgebung gegenüber und warnte vor einer Überschätzung: 
„Sie ist ferner deshalb irrig, weil die eigentliche soziale Missstimmung ihren Grund nicht sowohl in materiellen Missständen (die eigentlichen Träger derselben sind die bessergestellten Arbeiter) als in dem Drange nach wirklicher Rechtsgleichheit und Teilnahme an den Errungenschaften der modernen Kultur hat. Befriedigung kann für die unzufriedenen Arbeiterkreise nur geschaffen werden, wenn man ihnen das Gefühl wirklicher Gleichberechtigung mit den besitzenden Klassen [...] verschaffen kann. [...] Während die Regierung bis jetzt nur die Fürsorge für den arbeitsunfähigen Arbeiter umfasst, handelt es sich bei jenen Forderungen um die Verbesserung des gesunden und arbeitsfähigen Arbeiters, und schon darin liegt für alle Arbeiter, welche überhaupt so weit fortgeschritten sind, dass sie sich am öffentlichen Leben beteiligen, eine unendlich viel größere Zugkraft als in jener Fürsorge“" (Rothfels 1927: 74-75).

Ursächlich für die Unruhe unter den Arbeitern waren seines Erachtens nicht so sehr der materielle Mangel, sondern vielmehr das Bedürfnis nach faktischer Rechtsgleichheit. Die Versicherungsfrage traf damit nicht den Kern der sozialen Bewegung (Rosenstock 1934: 3-4). Wie bereits in einem vorausgegangenen Artikel zum vormodernen Allokationsprozess von Arbeit in Deutschland dargelegt (Pierenkemper/Kendzia 2010), stellte der eigentliche Beginn staatlicher Sozialpolitik das Regulierungsedikt von 1839 dar, welches Schutzbestimmungen für die Arbeiter beinhaltete. Den nachhaltigsten Eindruck mit Blick auf die staatliche Sozialpolitik hinterließ gleichwohl die Bismarcksche Sozialgesetzgebung. Bismarck beabsichtigte damit, den besitzlosen Klassen, die zahlenmäßig am stärksten innerhalb der Bevölkerung vertreten waren, zu zeigen, dass der Staat sowohl eine notwendige also auch wohltätige Institution ist. Im Kern zielte die soziale Versicherungsgesetzgebung darauf ab, die sozialdemokratische und revolutionäre Bewegung, die zunehmend an gesellschaftlichem Einfluss gewann, zu schwächen (Rosenstock 1934: 3). Bismarck, als der „weiße Revolutionär“ (Schmuhl 2003: 1), versuchte aus den Arbeitern seinen patriarchalischen Vorstellungen gemäß Staatsrentner beziehungsweise Staatsdiener zu machen - mit dem Unterschied, dass diese ihre Beschäftigung in der freien Wirtschaft nachgingen (Hentschel 1983: 25-26, Boeckh et al. 2004: 63). De Swaan schreibt hierzu:

„Es diente hauptsächlich der Staatsbildung, sollte ganz bewusst den neuen deutschen Staatsapparat stärken, eine industrielle Arbeiterklasse fest einbinden [...]. Bismarck plante eine Klasse von Staatspensionären: der Regierung loyal ergeben und streitbar gegen jede Veränderung, die ihre kleinen Vorteile hätte gefährden können - zwar ohne Vermögen, aber mit einem eigenen materiellen Interesse am Bestand der politischen Ordnung“" (de Swaan 1993: 208).

De Swaans Äußerung ist von großem Nutzen für die Untersuchung, zumal für die Bildung einer „Klasse von Staatspensionären“ einzig Elemente des Berufsbeamtentums in Frage kommen konnten. Inwieweit sich Bismarck daran orientierte, ist fraglich. Deutlich wird aber, dass sich Bismarck durch die soziale Absicherung seiner „Staatsdiener“, ähnlich wie beim Berufsbeamtentum, eine gewisse Treue, beziehungsweise Loyalität gegenüber dem Staat erhoffte durch eine modifizierte Form der Gehorsamspflicht der Beamten für die Lohnarbeiterschaft, ohne lebenslange Anstellung oder Laufbahnprinzip. 
Die in den Jahren von 1883 bis 1891 verabschiedeten Gesetze der Sozialversicherung in Deutschland beinhalteten im Kern folgende Strukturmerkmale:

- „Pflichtversicherung für Arbeiter und Angestellte bis zu einem Jahreseinkommen von 2.000 Mark

- Beitragsfinanzierung mit variierendem Anteil zwischen Arbeitgebern und Arbeitnehmern. Von einem Drittel zu zwei Drittel bei der GKV, eine paritätische Beitragsleistung bei der Invaliditäts- und Altersicherung und eine allein von den Unternehmern zu zahlende Unfallversicherung.

- $\quad$ staatlicher Zuschuss zur Gesetzlichen Rentenversicherung

- Beitrags-Leistungsbezug bei geldlichen Leistungen (Äquivalenzprinzip)

- Solidarprinzip bei Sach- und Dienstleistungen im Rahmen der KV

- Selbstverwaltung der Versicherungsträger

- Organisatorische Vielfalt in allen Versicherungszweigen

- Differenzierung der Sozialleistungen nach Maßgabe rechtlich normierter Anspruchsursachen (Kausalitätsprinzip) und nicht nach Maßgabe individuell oder sozial bestimmter Leistungszwecke (Finalitätsprinzip)““(Boeckh et al. 2004: 64-65).

Alber führt für die Erklärung der staatlichen Sozialpolitik funktionalistische und konflikttheoretische Modelle an. Nach Ersteren ist die Sozialgesetzgebung als Reaktion auf veränderte Arbeits- und Lebensformen im Zuge des Industrialisierungs- und Urbanisierungsprozesses zu verstehen, nach Letzteren als Folge von Demokratisierungsprozessen und der fehlenden Legitimität der politischen Elite (Alber 1982: 119).

\subsection{Die Krankenversicherung}

Die Krankenversicherung, welche ab dem 15. Juni 1883 galt, bezog sich auf Beschäftigte aus der Industrie, dem Handwerk, dem Handel, der Binnenschifffahrt und weiteren speziellen Dienstleistungsbetrieben bis zu einer bestimmten Einkommenshöhe. Die Beiträge entrichteten zu zwei Dritteln die Arbeiter und zu einem Drittel die Arbeitgeber und waren auf höchstens 6 $\%$ des Arbeitsverdienstes beschränkt. Die Ausweitung der Krankenversicherung auf Familienangehörige war nicht obligatorisch, jedoch grundsätzlich möglich. Eine Entschädigung für die ersten vier Wochen (später 13 Wochen) der Erwerbsunfähigkeit, die durch einen Unfall verursacht wurde, sollte nach dem Gesetzentwurf aus dem Jahr 1881 nicht mehr Gegenstand der Unfallversicherung, sondern der Krankenversicherung sein. Hierzu musste vor der Verabschiedung eines Gesetzes zur Unfallversicherung eine Prüfung des Krankenversicherungswesens erfolgen, weil nicht gewährleistet war, dass Arbeiter, die gegen Unfall versichert werden sollten, bereits auch gegen Krankheit versichert waren. Maßgeblich zur Entstehung der Krankenversicherung trug der bereits erwähnte Staatsbeamte Theodor Lohmann bei. Als Grundprinzip des 1883 beschlossenen Gesetzes galt die Durchführung des allgemeinen Krankenversicherungszwanges - freie Hilfskassen und Spezialkassen blieben zunächst neben den Zwangskassen erhalten. Da die Krankenversicherung versicherungstechnisch an die Ele- 
mente anknüpfte, die sich bereits bewährt hatten, war sie - mit Blick auf alle drei sozialen Versicherungszweige - am unproblematischsten einzuführen. Versicherungsträger der Krankenversicherung wurden Ortskrankenkassen. Voraussetzung hierfür war, dass mindestens 100 Beitragspflichtige ausfindig gemacht werden konnten. Wurde diese Anzahl unterschritten oder gehörten die Personen keiner Orts-, Betriebs, Bau- oder anderen Krankenkasse an, so mussten die Gemeinden eine eigene Krankenversicherung einrichten. Bei der Fertigstellung des Gesetzes 1883 wurden die Lohnarbeiter in der Land- und Forstwirtschaft vom Versicherungszwang ausgeklammert, obwohl diese häufig zwischen landwirtschaftlichen und gewerblichen Betrieben wechselten. Begründet wurde dieser Schritt damit, dass landwirtschaftliche Arbeiter eine Krankenversicherung nicht so nötig hätten wie gewerbliche Arbeiter, da ihnen bei einer Erkrankung in der Regel durch ihre Familie oder Nachbarn geholfen würde. Anders stellte sich eine derartige Situation innerhalb der industriellen Bevölkerung dar. Darüber hinaus sollte die häufig auf Naturalwirtschaft basierende Unterstützung des Arbeitgebers innerhalb der Landwirtschaft nicht durch ein auf der Geldwirtschaft beruhendes System der Krankenkassen verdrängt werden. Als weiteres Argument wurde angeführt, dass auf dem Land nur schwer zwischen Dienstboten (Gesindewesen) und landwirtschaftlichen Arbeitern differenziert werden konnte. Etwas später, mit dem Jahr 1886, wurden allerdings im Rahmen eines neuen Gesetzes auch die land- und forstwirtschaftlichen Arbeiter unter dem allgemeinen Krankenversicherungszwang zusammengefasst. Da beim Umzug in das Gebiet einer anderen Krankenkasse kein Eintrittsgeld erhoben werden durfte und keine Versicherungslücken entstanden, gewährte die organisierte soziale Krankenkasse ein enorme Freizügigkeit - Arbeitslosigkeit führte dennoch stets zu Versicherungslosigkeit und ein Wechsel der Arbeitsstätte brachte in der Regel auch einen Krankenkassenwechsel mit sich (Rosenstock 1934: 5-22).

Nach den ersten 13 Wochen der Versorgung der Unfallverletzten durch die Krankenversicherung erhielten die Arbeiter Hilfe aus der Unfallversicherung, weshalb Kranken- und Unfallversicherung seit ihrem Bestehen in einem engen Zusammenhang standen (Rosenstock 1934: 45). 


\subsection{Die Unfallversicherung}

Das zunächst 1871 verabschiedete Haftpflichtgesetz hatte den Nachteil, dass der Geschädigte in der Regel mit Hilfe eines Rechtsprozesses die Schuld des Arbeitgebers an dem Arbeitsunfall nachweisen musste. In der Folge machte sich eine generelle Unzufriedenheit mit dieser Regelung - auch auf der Seite der Unternehmer, die ohne staatliche Intervention eine Einschränkung ihrer Wettbewerbsfähigkeit befürchteten - breit (Butterwegge 2001: 28). Das Unfallversicherungsgesetz zielte zunächst im Kern auf Beschäftigte in Industriebetrieben ab. Unternehmer wurden auf eigene Kosten dazu verpflichtet, Arbeiter und Angestellten mit weniger als 2.000 Mark Jahreseinkommen in selbstverwalteten Berufsgenossenschaften gegen Unfälle abzusichern. Die nach der jeweiligen Branche unterschiedlichen Berufsgenossenschaften hatten den Verunglückten oder ihren Hinterbliebenen nach Ablauf der gesetzlichen Krankenfürsorge eine verdienstbezogene Rente auszuzahlen. Zugleich durften die Berufsgenossenschaften Vorschriften zur Vermeidung von Unfällen einführen (Alber 1982: 68, Molitor 1987: 88).

Bereits seit einiger Zeit existierten Einrichtungen, die es den Arbeitern ermöglichten, sich gegen die wirtschaftlichen Folgen von Betriebsunfällen abzusichern - so z. B. seit 1870 ein Unfallversicherungsverein der Rheinischen Erzgruben und Hüttenwerke und andere Einrichtungen. Des Weiteren gab es schon private Unfallversicherungsgesellschaften bei denen man sich gegen die wirtschaftlichen Folgen von Unfällen absichern konnte. Nach der Reichsunfallstatistik von 1882 waren in dem Jahr noch 50\% aller Arbeit gegen Unfälle nicht versichert. Die Unzufriedenheit über das Haftpflichtgesetz von 1871 sowohl auf Seiten der Arbeitgeber als auch der Arbeitnehmer, wurde immer häufiger als Anlass genommen, gegen das bestehende System zu agitieren. Aufgrund ähnlicher Schwierigkeiten bei der Abgrenzung zwischen landwirtschaftlicher und gewerblicher Arbeit blieben die Arbeiter in land- und forstwirtschaftlichen Betrieben zunächst von der Unfallversicherungspflicht ausgenommen. Im Jahr 1886 aber wurde das Gesetz auch auf land- und forstwirtschaftliche Betriebe ausgedehnt. Handwerksbetriebe blieben dennoch von der Regelung ausgenommen, da sich in deren Reihen nur selten Unfälle ereigneten und ein Großteil dieser Art von Betrieben lediglich mit einem Gehilfen arbeitete. Als Versicherungsträger wurden Berufsgenossenschaften mit Selbstverwaltung für bestimmte Bezirke errichtet. Laut Unfallversicherungsgesetz von 1884 erhielten Unfallverletzte die Kosten des Heilverfahrens, Vollrenten in Höhe von $662 / 3 \%$ des Jahresarbeitsverdienstes oder Teilrenten bei teilweiser Erwerbsunfähigkeit ( $§ 5)$. Hinzu kamen unter anderen die Erstattung der Beerdigungskosten (mindestens 30 Mark) im Falle der Tötung, eine Hin- 
terbliebenenrente an die Witwe in Höhe von $20 \%$, an die vaterlosen Kinder $15 \%$, an die Vollwaisen 20\% des Jahresarbeitsverdienstes des Getöteten (Rosenstock 1934: 23-35).

\title{
4.3 Die Alters- und Invaliditätsversicherung
}

Unter dem Begriff der Invalidität ist generell die „physisch oder psychisch verursachte, dauerhafte Minderung des Leistungsvermögens während des Erwerbsalters gemeint, die bis zur vollständigen Erwerbsunfähigkeit gehen“ (Molitor 1987: 93) kann. Lohmann kritisierte an dem Entwurf der Alters- und Invalidenversicherung, dass erst ein neuartiger Versicherungsträger bürokratischer Art, die Landesversicherungsanstalten, geschaffen werden mussten. Des Weiteren verhinderte das Umlageverfahren seiner Meinung nach die Aufstellung einer soliden Bilanz (Rothfels 1927: 81). Aus den Verhandlungen des Reichstags gehen die Gründe für die Einführung der Bismarckschen Invaliditäts- und Altersversicherung hervor:

\begin{abstract}
„Erfahrungsgemäß wird durch die Erreichung eines hohen Lebensalters in den meisten Fällen eine größere oder geringere Beschränkung der Erwerbsfähigkeit bedingt. Wo diese Voraussetzung zutrifft, bildet die Altersrente einen Zuschuss zu dem noch vorhandenen Arbeitsverdienst. Dieser Zuschuss wird es dem altgewordenen Arbeiter erleichtern, eine Überanstrengung der ihm verbliebenen Arbeitskraft zu vermeiden und sich den Rest seiner Arbeitsfähigkeit länger zu erhalten, den Eintritt der völligen Erwerbsunfähigkeit also hinausschieben. Freilich darf die in diesem Falle zu gewährende Leistung den Höchstbetrag der an Invalide zu gewährenden Rente nicht erreichen, weil sonst der Anlass, die verbliebene Arbeitsfähigkeit weiter auszunutzen, fortfallen würde“ (Molitor 1987: 27).
\end{abstract}

Zuvor sorgten rechtliche Verpflichtungen oder emotionale Bindungen für die Grundversorgung von Menschen, die z. B. durch Alter nicht oder nur bedingt in der Lage waren ihren Lebensunterhalt aus eigener Kraft zu bestreiten. Kinder, Greise, sowie Kranke und Behinderte waren im Schoße der Familie vor den größten Risiken des Lebens geschützt (Butterwegge 2001: 56-57).

Bisher hatten noch keine gesetzlichen Einrichtungen bestanden, die den Arbeitern Schutz und Fürsorge im Fall der Invalidität und Altersschwäche garantierten. Zumeist war die Bevölkerung auf die Armenpflege angewiesen. Einzig die auf genossenschaftlicher Vereinigung beruhenden Knappschaftskassen bildeten eine Ausnahme. Zusätzlich existierten noch einzelne Invalidenkassen, die freiwillig von Betrieben gegründet wurden. Bei der Errichtung der Invaliditäts- und Altersversicherung ließ man sich von den bereits bestehenden Knappschaftskasseneinrichtungen inspirieren, die man anschließend verbesserte. Übernommen wurden von den Knappschaftseinrichtungen im Invaliditätsversicherungsgesetz unter anderem der Versicherungszwang, die Beitragsbeteiligung der Arbeitgeber und Arbeitnehmer, die Verpflichtung der Arbeitgeber auch für die Einziehung und Abführung der Beiträge ihrer 
Arbeiter aufzukommen, die Anmeldung der neu eingestellten Arbeitnehmer durch die Arbeitgeber sowie die gemeinsame Verwaltung der Organisation durch Arbeitnehmer- und Arbeitgebervertreter (Rosenstock 1934: 46, 62). Nach dem Invaliditäts- und Alterssicherungsgesetz waren alle Arbeiter vom 16. Lebensjahr an versicherungspflichtig. Die Mittel der Versicherung wurden durch einen Reichszuschuss sowie durch gleich hohe Beiträge der Arbeitgeber und der Versicherten bewerkstelligt. Der relativ niedrige und rechtlich komplexe Rentenanspruch kam zustande, wenn beim Versicherten Erwerbsunfähigkeit festgestellt wurde und dieser über einen langen Zeitraum (5-30 Beitragsjahre) hinweg Beiträge entrichtet hatte (Alber 1982: 54-55).

Tabelle 5: Beispielrechnung der jährlichen Beiträge für die einzelnen Versicherungen ${ }^{1}$

\begin{tabular}{|l|c|c|c|}
\hline & \multicolumn{3}{|c|}{ Jährlicher Beitrag im Durchschnitt für jeden Versicherten seitens: } \\
\hline Art der Versicherung & des Arbeitgebers & des Versicherten & Beide zusammen \\
\hline & \multicolumn{3}{|c|}{ in Mark } \\
\hline Krankenversicherung & 4,40 & 8,80 & 13,20 \\
\hline Unfallversicherung & 14,46 & - & 14,46 \\
\hline $\begin{array}{l}\text { Alters- und Invali- } \\
\text { denversicherung }\end{array}$ & 4,00 & 4,00 & 8,00 \\
\hline Zusammen & 22,86 & 12,80 & 35,62 \\
\hline
\end{tabular}

Quelle: Rosenstock 1934: 80.

Die gesetzliche Altersgrenze für die Inanspruchnahme der Altersrente betrug zur Zeit Bismarcks 70 Jahre (Molitor 1987: 28). Die staatlichen Sozialleistungen implizierten, wenn auch nicht für alle Arbeiter und Arbeitnehmer zu der Zeit, gewissermaßen eine Abschwächung arbeitsmarktlicher Zwänge (Vobruba 1978). Ähnlich argumentiert Rimlinger, nach dessen Auffassung im Zuge der Auflösung traditioneller Schutzverbände und der Expansion des Arbeitsmarktes die Frage der Einkommenssicherung und die gesellschaftliche Integration vermehrt in den Vordergrund rückte (Rimlinger 1971). Die soziale Sicherung diente dazu, die Risiken der faktoranbietenden privaten Haushalte, die unvermeidlich mit der wirtschaftlichen

\footnotetext{
${ }^{1}$ Bei der Invaliditätsversicherung kam noch der Reichsbetrag (50 Mark Zuschuss zu jeder Rente) hinzu. Die Land- und Forstwirtschaft ist bei der Tabelle nicht in Betracht gezogen worden (Rosenstock 1934: FN 308).
} 
Dynamik einher gehen, langfristig und unabhängig vom konjunkturellen Verlauf abzufedern (Molitor 1987: 205). Die Bismarckschen Sozialversicherungssysteme hatten einerseits durch die Versicherungspflicht bereits frühzeitig einen breiten Mitgliederstamm, andererseits wurden gut Verdienende und Selbstständige zumindest staatlich nicht abgesichert (Alber 1982: 146). Die Invalidenversicherung gewährte jedoch nur für wenige nicht mehr arbeitsfähige Arbeiter (ein Rentenbezug war erst ab dem siebzigsten Lebensjahr möglich) einen Zuschuss zum Unterhalt „am billigsten Orte“ und ist nicht als Pendant zu einem heutigen Ruhegehalt aufzufassen. Das zugrunde liegende Kapitaldeckungsverfahrens erforderte eine lange Anwartschaft (Pierenkemper 2006a :45-46).

\subsection{Die Rentenversicherung}

Im Jahr 1891 wurde in Deutschland die Rentenversicherung eingeführt. Als Vorläufer fungierte die zuvor erwähnte Invaliditäts- und Altersversicherung. Ihre Mittel setzten sich zu je einem Drittel aus den Beiträgen der Arbeiter, der Arbeitgeber und staatlichen Zuschüssen zusammen. Versicherungspflicht bestand auch hier nur für Arbeiter mit einem Jahreseinkommen von unter 2.000 RM (Pohl 1979: 205-206). Seit 1911 war sie zugleich auch eine Versicherung der Hinterbliebenen. Nur zwei Jahre später folgte durch das so genannte Angestelltenversicherungsgesetz (AVG) eine Rentenversicherung für die Angestellten. Für die im Bergbau beschäftigten Arbeiter waren nach wie vor die Knappschaften zuständig (Grohmann 1965: 30). Allgemein wird unter einer Rente die regelmäßige Zahlung eines Einkommens aufgefasst, das auf einem rechtlichen Anspruch basiert und entweder aus Kapitalerträgen oder Beiträgen resultiert. Die Renten wurden in dem besagten Zeitraum allerdings erst ab einem Alter von 70 Jahren ausgezahlt - bei einer durchschnittlichen Lebenserwartung im Jahr 1910 von 47,4 Jahren bei Männern und 50,7 Jahren bei Frauen (Winkels 2007: 18). 
Tabelle 6: Mitgliederzahl der Sozialversicherung von 1890 bis 1913 (in Millionen)

\begin{tabular}{|c|c|c|c|}
\hline Jahr & Unfallversicherung & Krankenversicherung & $\begin{array}{c}\text { Alters-und Invali- } \\
\text { denversicherung }\end{array}$ \\
\hline 1890 & 13,7 & 6,6 & - \\
1895 & 18,4 & 7,5 & 11,8 \\
1900 & 18,9 & 9,5 & - \\
1905 & 18,7 & 11,9 & 13,9 \\
1910 & 24,2 & 14 & 15,7 \\
1913 & 25,8 & 14,6 & 16,3 \\
\hline
\end{tabular}

Quelle: Kuczynski 1947: 228.

Abschließend lässt sich konstatieren, dass im Zuge der staatlichen Sozialpolitik nach Bismarck die Lohnarbeit insgesamt aufgewertet wurde. Lohnarbeiter wurden als potentielle Leistungsempfänger bestimmter Versicherungsleistungen eingestuft, wohingegen Erwerbslose nach wie vor Leistungen der Armenfürsorge erhielten (Butterwegge 2001: 29). Ihrem Wesen nach war die Bismarcksche Sozialpolitik vor allem Staatspolitik (Rothfels 1970: 169), die nicht unter einer spezifisch sozialen Zielsetzung stand. Mit den patriarchalischen Verhältnisse in Ostelbien war Bismarck seit jeher vertraut, weshalb die dortigen Gepflogenheiten in Bismarcks Sozialpolitik erkennbar blieben (Rothfels 1929: 6-7). Die soziale Frage konnte nach Auffassung Bismarcks nur der Staat bewältigen. Im Jahr 1885 schrieb von Rottenburg, ein Mitarbeiter Bismarcks: „Gewiss kann der Einzelne viel Gutes tun, aber die soziale Frage lösen kann nur der Staat“ (Rothfels 1929: 8).

Tabelle 7: Gesamtbeiträge zur Sozialversicherung (in Millionen Mark) 1887-1913

\begin{tabular}{|c|c|}
\hline Jahr & Gesamtbeiträge \\
\hline 1887 & 98,6 \\
1900 & 485,7 \\
1913 & 1158,1 \\
\hline
\end{tabular}

Quelle: Kuczynski 1947: 230.

Die Unfallversicherung umfasste anfangs etwa $17 \%$ der Erwerbsbevölkerung. Nach Erweiterung der Versicherungspflicht waren im Jahr 1890 schon zwei Drittel der Erwerbsbevölkerung eingebunden. Die Krankenversicherung hatte zu Beginn im Jahre 1885 einen Aus- 
dehnungsgrad von $22 \%$ und dehnte sich bis 1890 auf gut ein Drittel der Erwerbsbevölkerung aus. Die Rentenversicherung schloss schon zu Beginn mehr als die Hälfte der Erwerbstätigen ein (Alber 1982: 147).

Die Sozialversicherungen konnten allerdings ihre positive Wirkung erst nach einer gewissen Zeit unter Beweis stellen. Sie bildeten eine Institution zwischen Staat und Gesellschaft, die sich dadurch auszeichnete, dass sie staatsnah, selbstverwaltet und im Wesentlichen durch Beiträge finanziert wurde (Stolleis 2001: 255, 265). Durch die Institutionalisierung der Sozialversicherungssysteme schuf Bismarck „den institutionellen Kern des Wohlfahrtsstaates“ (Alber 1982: 19).

Zwar erwähnt Rothfels in seiner 1929 gehaltenen Rede über die Prinzipienfragen der Bismarckschen Sozialpolitik einen „Normalarbeitstag“ innerhalb eines Aktenstücks aus dem Jahr 1885 (Rothfels 1929: 19), von einem Normalarbeitsverhältnis in der heutigen Ausgestaltung kann gegen Ende des 19. Jahrhunderts allerdings noch keine Rede sein. Dennoch gilt es festzuhalten, dass mit der Einführung der Bismarckschen Sozialversicherung die Grundzüge des heutigen Normalarbeitsverhältnisses bereits erkennbar wurden. Folgt man der Minimaldefinition des Normalarbeitsverhältnisses als eine abhängige und sozialversicherungspflichtige Vollzeitbeschäftigung, so lässt sich argumentieren, dass in der zweiten Hälfte des 19. Jahrhunderts der Kern des Normalarbeitsverhältnisses geschaffen wurde. Auch Butterwegge argumentiert, dass mit der Bismarckschen Sozialgesetzgebung das Lohnarbeitsverhältnis einen großen Schritt in Richtung Normalarbeitsverhältnis unternahm (Butterwegge 2001: 54). 


\section{Entwicklung der Sozialpolitik von 1890 bis 1914}

Während der Jahre von 1890 bis 1914 veränderte sich die politische Landschaft in Deutschland gewaltig. Am 1. Oktober 1890 lief das so genannte Sozialistengesetz nach zwölf Jahren aus und die Sozialdemokratische Partei wuchs bis 1912 mit 34,8\% der Stimmen zur stärksten Partei an. Damit einher ging auch der Niedergang der nationalliberalen und konservativen Parteien, was den Abtritt Bismarcks von der politischen Bühne 1890 zur Folge hatte (Schildberg-Schroth: 87).

Angesichts eines derartigen politischen Wandels innerhalb der Gesellschaft ist es interessant zu betrachten, wie sich die Tarifvertragsparteien zum damaligen Zeitraum verhielten. In Deutschland wurde der erste Tarifvertrag 1873 eingeführt - vom Staat jedoch noch nicht akzeptiert. Erst ab 1899 breitete sich der Tarifvertrag in Deutschland und in anderen westeuropäischen Staaten aus (Ullmann 1977, Engelberger 1995). Vor dem Ersten Weltkrieg existierten bereits 12.369 Tarifverträge für rund 193.000 Betriebe mit 1,8 Millionen Beschäftigten (Hueck et al. 1963: 12). In einer kurzen Episode spielte die Arbeiterschutzgesetzgebung wieder eine gewisse Rolle. Es kam zu der sozialpolitischen Reformära Berlepsch, welche Pflichten zum Erlass von Fabrikordnungen mit sich brachten, die im Wesentlichen auf die Verbesserung von Arbeitsabläufen innerhalb der Betriebe abzielten (von Berlepsch 1987, Syrup 1957).

Durch das Gesetz betreffend die Abänderung der Gewerbeordnung (GewO), beziehungsweise das Arbeiterschutzgesetz vom 1. Juni 1891 wurde ein Beschäftigungsverbot für schulpflichtige Kinder erwirkt, der 10-Stunden-Arbeitstag für Jugendliche und der 11-stündige Arbeitstag bei Frauen eingeführt sowie die rechtliche Möglichkeit geschaffen, Arbeiterausschüsse in Fabrikbetrieben zu bilden - was jedoch von keiner großen Arbeitgeberschaft mitgetragen wurde (Förster 2003: 183-184). Zusätzlich beinhaltete das Arbeiterschutzgesetz eine Verschärfung des Truckverbots (Verbot der Entlohnung von Arbeitnehmern durch Waren), das Verbot der Sonntagsarbeit sowie das Verbot der Nachtarbeit für Frauen und Jugendliche unter 16 Jahren. Ergänzt wurde das Gesetz durch das „Reichsgesetz betreffend Kinderarbeit in gewerblichen Betrieben“ vom 30. März 1903. Mit dem Hausarbeitsgesetz vom 20. Dezember 1911 wurden Vorschriften zum Gesundheitsschutz und der Arbeitssicherheit der Heimarbeiter erlassen. Zwischen 1890 und 1900 setzte der Staat zwar auf Repression gegen die Arbeiterbewegung, konnte aber nicht verhindern, dass die Zahl der organisierten Arbeiter von 280.000 auf 680.000 wuchs, bis sie 1914 schließlich 2,5 Millionen Arbeiter erreichte. Zugleich formierten sich die Arbeitgeber in Verbänden, was zur Folge hatte, dass gegen Ende des 19. 
Jahrhunderts eine Begegnung auf Verbandsebene zwischen Arbeitgebern und Arbeitnehmern möglich wurde. Gleichwohl gewährte der Staat bis 1914 beiden Parteien keine rechtlich fixierten Mitwirkungsmöglichkeiten und erkannte Tarifverträge bis dato nicht an, obgleich das Reichsgericht 1910 beschlossen hatte, Tarifverträge als bindend auszulegen. Im Zuge des Reichsvereinigungsgesetz vom 19. April 1908 durften nun auch Frauen und Jugendlichen solchen Verbänden beitreten und auch der Zusammenschluss der Landarbeiter wurde erlaubt (Stolleis 2001: 258-260).

Im Jahr 1911 wurden die drei zunächst getrennten Zweige der Sozialversicherung in der Reichsversicherungsverordnung (RVO) zusammengefasst, die bis in die 1970er Jahre Bestand hatte und dann später von dem Sozialgesetzbuch abgelöst wurde. Zusammen mit der RVO wurde 1911 das Angestelltenversicherungsgesetz beschlossen, das die freiwilligen Hilfskassen in Ersatzkassen der Gesetzlichen Krankenversicherung überführte. Gleichzeitig wurden Witwenrente und Angestelltenversicherung nach dem Muster der Arbeiterrentenversicherung eingeführt. Diese bezog alle Angestellten mit einem Jahreseinkommen zwischen 2.000 und 5.000 Mark mit ein (Boeckh et al. 2004: 66-67, Rother 1994: 229). Mit der Einführung der Angestelltenversicherung positionierten sich die Angestellten zwischen den Arbeitern und den Beamten als eigenständige Gruppe innerhalb der deutschen Gesellschaft (Croner 1962). Die Ausstattung der Angestelltenversicherung mit höheren Leistungen sowie höheren Beiträge sollte dazu dienen, die Angestellten ,gegen die Agitation der Sozialdemokratie und der sozialistischen Freien Gewerkschaften zu immunisieren und für den Staat bzw. die eigene Partei zu gewinnen“" (Ritter 1998: 94-95). Die zweieinhalb Jahrzehnte zwischen dem Abdanken Bismarcks und dem Ersten Weltkrieg waren sowohl durch den weitergehenden Strukturwandel als auch durch eine sich etablierende Angestelltenschaft geprägt.

An den Zahlen des Statistischen Bundesamtes lässt sich erkennen, dass mit der Zeit der Anteil der Selbständigen deutlich abnahm, allerdings durch den Anstieg der mithelfenden Familienangehörigen abgemildert wurde. Das bedeutet, dass die Zahl der Erwerbspersonen, die keiner abhängigen Beschäftigung nachgingen, im Grunde weitestgehend stabil blieb. Dies gilt auch für den Anteil der abhängig Beschäftigten. Auffallend hingegen ist das enorme Wachstum der Angestellten und Beamten. Dies lässt durch die Expansion industrieller Großunternehmen seit etwa 1870 und der Umstellung der Betriebsführung von Eignern zu Managern erklären. Mehr noch als im Handel ermöglichte damit die Hochkonjunktur gewerblichindustrieller Unternehmen und das damit verbundene Wachstum der Betriebsgrößen die Entstehung zahlreicher neuer Berufe oder Positionen für die Angestelltenschaft (Schulz 2000: 5,12). Interpretiert man diese Zahlen, so lässt sich sagen, das insbesondere die Selbständigen 
und mithelfenden Familienangehörigen in der Landwirtschaft vorzufinden sind, wohingegen die Arbeiter im gewerblichen Bereich, die Angestellten im Bereich der Dienstleistungen anzusiedeln sind. Dennoch ist darauf zu verweisen, dass die Zuordnung einzelner Erwerbstätiger auf die Berufsgruppen im Sprachgebrauch stark variierte und demzufolge eine eindeutige $\mathrm{Zu}$ rechnung aus heutiger Sicht unsicher bleibt. Private und öffentliche Beamte wurden beispielsweise nur unzureichend voneinander unterschieden. Zu den (Privat-)Beamten gehörten seinerzeit Kassierer, Buchhalter sowie Betriebsleiter. Bis 1925 zählten leitende Angestellte zu den Selbständigen, dagegen wurden Verkäuferinnen und Ladendiener zu den unteren Beamten gezählt (Schulz 2000: 53).

Abbildung 2: Berufsgruppen nach Berufszählungen

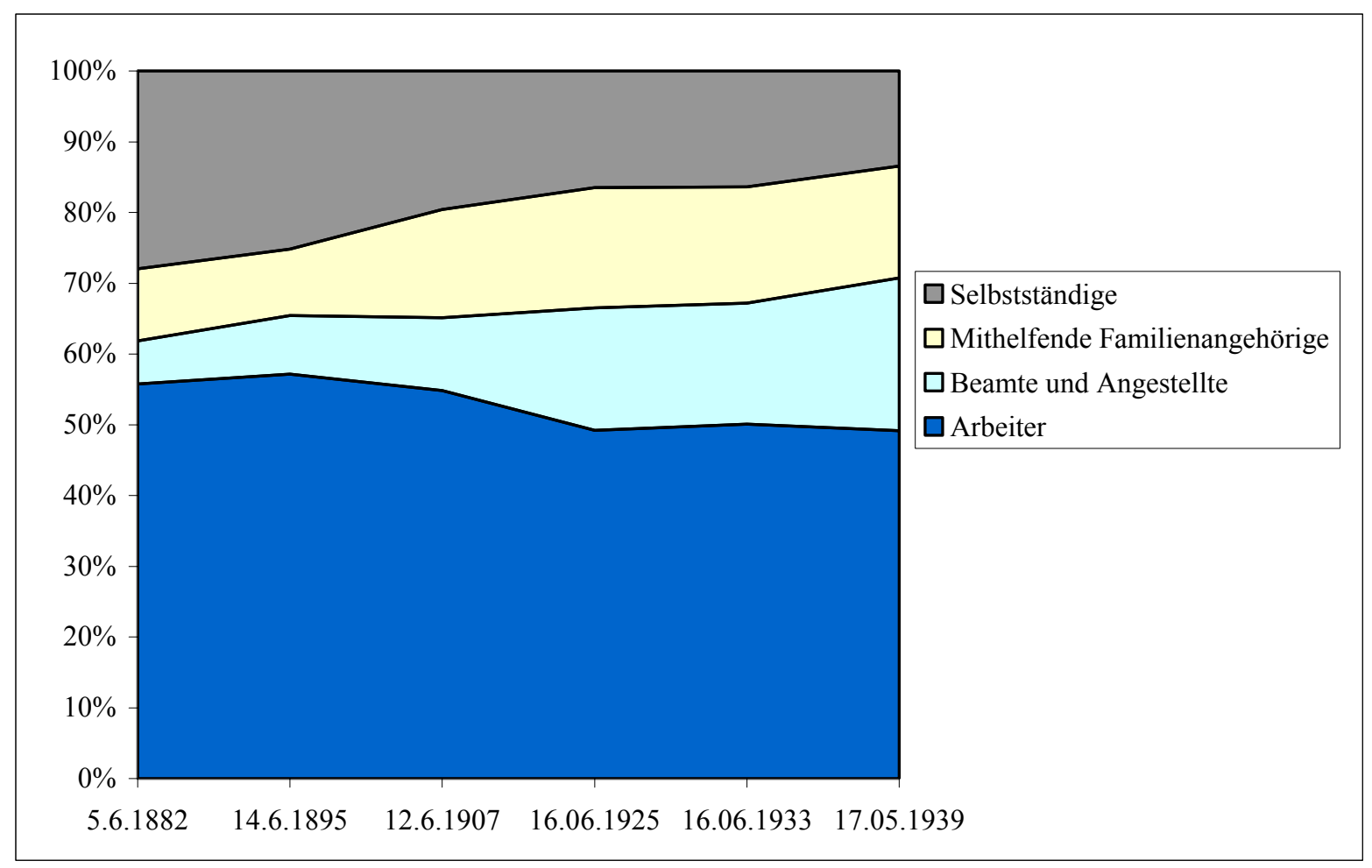

Quelle: Statistisches Bundesamt 1972: 142.

Im Zuge der Urbanisierung erfolgte ein quantitativer Ausbau der städtischen Verwaltungsapparate, welcher die Zahl der Beamten ebenso wie die Zahl der Angestellten rapide ansteigen ließ. So beschäftigte z. B. die Stadt Essen im Jahr 1840 acht und im Jahr 1900 bereits 377 halbamtliche Beamte (Gall 1990: 33). Eine erste gesetzliche Regelung, die sich dem Thema Kündigungsfrist annahm, beinhaltete das Allgemeine Deutsche Handelsgesetzbuch von 1861. Die Regelung bezog sich auf kaufmännische Angestellte und verlangte, dass ihr Arbeitsverhältnis nur mit einer Frist von sechs Wochen (zum Ende des jeweiligen Quartals) aufgelöst werden konnte. Erst im Zuge des bereits erwähnten Arbeiterschutzgeset- 
zes von 1891 wurde diese Regel auf Angestellte im technischen Bereich ausgeweitet. Hierbei handelte sich aber um ,abdingbares“, also nicht zwingendes Recht, so dass einzelvertraglich andere (kürzere) Kündigungsfristen möglich waren. Somit wurden die Angestellten zunächst, vor den Bismarckschen Sozialreformen, besser gestellt als die überwiegende Mehrheit der Arbeiter. Denn in der Regel erhielten Arbeiter keine Lohnfortzahlung im Krankheitsfall und konnte jederzeit, allenfalls mit ein- bis zweiwöchiger Frist, gekündigt werden (Schulz 2000: 15). Noch um 1900 waren ausgiebiger Absentismus (individuelles Fernbleiben von der Arbeit) und Fluktuationsraten von 80 bis $100 \%$ bei den Industriearbeitern innerhalb eines Jahres keine ungewöhnlichen Phänomene des Arbeitsalltags (Ritter/Tenfelde 1992, Tenfelde 1986). Die geschilderten Tendenzen in der Entwicklung des deutschen Arbeitsmarktes im 19. Jahrhundert lassen sich, soweit quantitative Daten für Arbeitslosigkeit, Arbeitszeit und Löhne zur Verfügung stehen, anschaulich in einer Grafik darstellen. Die Trends zu steigenden Löhnen und sinkender Arbeitszeit bei recht stabiler Ausschöpfung des Arbeitspotentials sind unübersehbar (Pierenkemper 2006b: 255).

Abbildung 3: Arbeitszeit, Entlohnung und Arbeitslosigkeit 1820 bis 1913

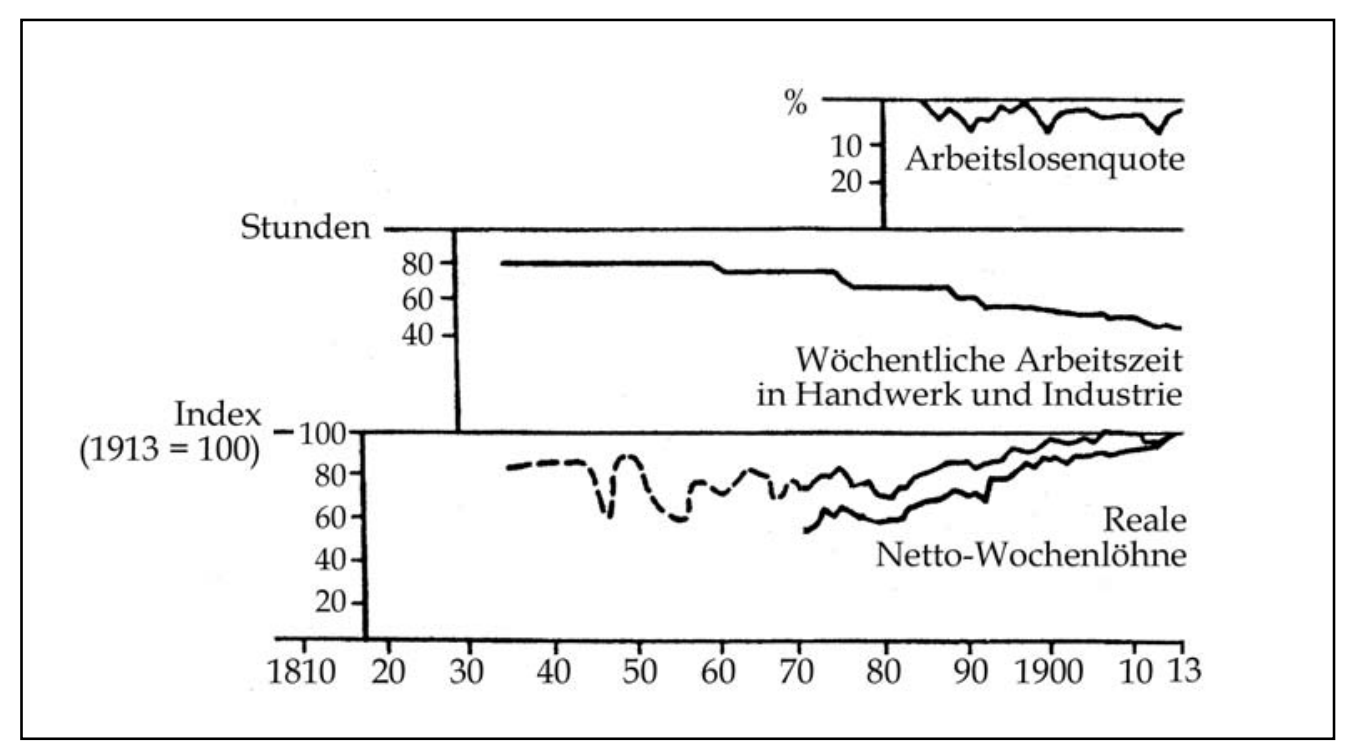

Quelle: Beschäftigung und Arbeitsmarkt: 255.

Abschließend lässt sich sagen, dass das Lohnarbeitsverhältnis bis 1914 einer Reihe von Veränderungen unterworfen war. Ähnlich argumentiert Schmuhl, der angibt, dass sich am Vorabend des Ersten Weltkriegs bereits die Grundmuster gebildet hätten, ,die in unserer Gesellschaft bis heute wirksam sind“ (Schmuhl 2003: 1). Damit ist vor allem der Zeitraum 
von den 1880er Jahren bis 1914 als eine äußerst prägende Epoche für das Lohnarbeitsverhältnis im Allgemeinen und für das Normalarbeitsverhältnis im Besonderen aufzufassen.

Aufgrund des bereits vorhandenen Arbeitsmarktes, seiner zunehmenden Dynamisierung durch die Industrialisierung und der weiten Verbreitung abhängiger Beschäftigungsverhältnisse waren die Lohnarbeiter in Phasen des wirtschaftlichen Abschwungs entweder vormodernen Formen der Erwerbstätigkeit oder aber der Erwerbslosigkeit ausgesetzt (Faust 1984: 217). Arbeitslosigkeit tauchte als Bezeichnung für einen sozialen Status erst Ende des 19. Jahrhunderts auf (Zimmermann 2006). Sie stellte seit den 1880er Jahren, Ausnahmen bildeten die Jahre 1892 und 1901, mit Arbeitslosenquoten von unter 5\% kein allzu drängendes gesellschaftliches Problem dar. Eine staatliche Zwangsversicherung gegen Arbeitslosigkeit entstand erst nach dem Ersten Weltkrieg. Zuvor setzte man auf die Arbeitsvermittlung, zumal die Kommunen hier bereits über Erfahrungen verfügten. Die niedrige Arbeitslosigkeit bis 1913 lässt sich zum einen durch eine stabile Nutzung des Erwerbspotentials erklären (Pierenkemper 2009: 15-20), zum anderen erscheint es möglich, dass - im Gegensatz zur Armut - Arbeitslosigkeit noch weitestgehend als individuell verschuldet angesehen wurde. 


\section{Schlussbemerkung}

In der vorliegenden Untersuchung wurde aus einem polit-ökonomischen Ansatz heraus veranschaulicht, welchen Einflüssen das Lohnarbeitsverhältnis von seiner Etablierung und Durchsetzung im 19. Jahrhundert bis zum Ausbruch des Ersten Weltkrieges unterlag. Hierbei stand in erster Linie die Ausgestaltung des Arbeitsverhältnisses im Hinblick auf das heutige Normalarbeitsverhältnis im Mittelpunkt.

Es konnte nachgewiesen werden, dass der betrachtete Zeitraum von einem enormen Bevölkerungswachstum und einer damit einhergehenden rasanten Verbreitung der Erwerbsarbeit geprägt war. Der weiter voranschreitende Strukturwandel führte zu einem Anstieg und einer Ausdifferenzierung der abhängigen Beschäftigungsverhältnisse: Neben der Industriearbeiterschaft konnte sich eine Angestelltenschaft etablieren. Außerdem stieg die Zahl der privaten und öffentlichen Beamten beträchtlich an. Zum einen wurde dies darauf zurückgeführt, dass sich die privaten Beamten aus leitenden Angestellten und anderen (nicht näher zu spezifizierenden) Berufsgruppen zusammensetzten. Zum anderen entstanden infolge des Urbanisierungsprozesses komplexe städtische Verwaltungsapparate, für die zahlreiche öffentliche Beamte benötigt wurden.

Bei den Überlegungen zur Einführung der Sozialversicherungen wurde deutlich, dass Arbeiter in der Landwirtschaft häufig noch in Naturalien anstatt in barem Lohn bezahlt wurden. Infolge der Urbanisierung und (Hoch-)Industrialisierung setzte sich die Lohnarbeit gegenüber alternativen Formen der gesellschaftlichen Organisation von Arbeit durch. Die aus der feudalen Abhängigkeit befreiten Arbeiter sahen sich vielen neuen Herausforderungen ausgesetzt, die unter dem Begriff der „Sozialen Fragen“ subsumiert wurden - wie seinerzeit z. B. durch Gustav Schmoller. Neben den Fragen nach einer Versorgung im Krankheitsfall und im Alter drängte sich vor allem eine Absicherung der Industriearbeiterschaft im Falle von Unfällen im Betrieb auf.

Frühe Initiativen qualifizierte Arbeiter an den Betrieb zu binden, wurden durch Unternehmer angestoßen, um die Qualität von Arbeitsprozessen gegen die hohen Fluktuations- und Absentismusraten zu schützen. Es konnte gezeigt werden, dass bereits eine Reihe von Unterstützungskassen bestanden, die über Beiträge der Arbeiter und Arbeitgeber finanziert wurden. Diese schlossen zunächst nur einen Teil der Arbeiterschaft ein und sicherten die Betroffenen auch nur notdürftig ab. Dennoch bargen diese Kassen bereits die wesentlichen Strukturelemente der späteren Bismarckschen Sozialversicherungen. Hierzu gehörten die Beitragsfinanzierung sozialer Versicherungsleistungen, aus der sich ein Rechtsanspruch auf den Bezug 
ableitete, der solidarische Ausgleich bei sozialen Risiken sowie die Selbstverwaltung der Organisation durch die Versicherten.

Die Institutionalisierung der Sozialversicherungen durch Bismarck sollte in erster Linie eine weitere Stärkung und Ausdehnung der Arbeiterbewegung verhindern. Seines Erachtens ließ sich die Soziale Frage allein durch den von ihm 1871 gegründeten Staat lösen. Bismarck beabsichtigte, mit Hilfe der sozialen Absicherung („Zuckerbrot“") aus der Lohnarbeiterschaft loyale Staatsrentner („Peitsche“) schaffen zu können.

Bei der Ausgestaltung des abhängigen Beschäftigungsverhältnisses durch die Sozialversicherungen konnte eine gewisse Analogie zum Berufsbeamtenverhältnis ausgemacht werden. Letzteres zeichnete sich durch Treue beziehungsweise Gehorsam gegenüber dem Staat und einer im Gegenzug dafür unbefristeten, unkündbaren und somit privilegierten Anstellung aus.

Mit dieser - auch heute noch erkennbaren - Struktur des Lohnarbeitsverhältnisses wurde im Kern das Normalarbeitsverhältnis gegründet. Insbesondere zum Ende des betrachteten Zeitraums lässt sich aufgrund der Abhängigkeit des Lohnarbeiters vom Arbeitgeber und die an die Erwerbsarbeit gekoppelten Beiträge ein abhängiges und sozialversicherungspflichtiges Vollzeiterwerbsverhältnis erkennen.

Verschiedene Arbeiterschutzgesetze und eine Regulierung der Arbeitszeit verbesserten vor dem Ersten Weltkrieg die Stellung der Lohnarbeiter auf dem Arbeitsmarkt. Neben der Arbeiterbewegung und ihren Selbsthilfeeinrichtungen sowie unternehmerischen Initiativen, trugen auch Intellektuelle wie beispielsweise der Verein für Socialpolitik zur Verbesserung der Lage der Arbeiter bei. Gesetzliche und sozialversicherungsrechtliche Regelungen begannen den Arbeitsmarkt zu regulieren und definierten erste Wesenszüge eines Normalarbeitsverhältnisses, obwohl vor dem Ersten Weltkrieg weder Arbeitslosenversicherung noch Kündigungsschutz existierten. Wenn wir also heute vom Normalarbeitsverhältnis sprechen, dann von dem in der Ausgestaltung Ende der 70er Anfang der 80er Jahre des 20. Jahrhunderts inklusive Arbeitslosenversicherung und Kündigungsschutz. Möchte man ein Bild für den $\mathrm{Zu}-$ stand des Normalarbeitsverhältnisses zum Ende des Betrachtungszeitraums benennen, so bestanden nach Bismarck bereits ein solides Fundament und mehrere Säulen eines Tempels. An seiner Fertigstellung wurde bis in die 70er und 80er Jahre des 20. Jahrhunderts weitergearbeitet. 


\section{Literaturverzeichnis}

Alber, Jens: Vom Armenhaus zum Wohlfahrtsstaat. Analysen zur Entwicklung der Sozialversicherung in Westeuropa, Frankfurt am Main 1982

Becker, Martin: Arbeitsvertrag und Arbeitsverhältnis während der Weimarer Republik und in der Zeit des Nationalsozialismus, Juristische Abhandlungen, Band 44, Frankfurt am Main 2005

Boeck, Jürgen et al.: Sozialpolitik in Deutschland, Wiesbaden 2004

Boehmer, Georg Ludwig: Principia iuris feudalis praesertim Langobardici quod per Germaniam obtinet, 5. Aufl., Göttingen 1789

Borchardt, Knut: Grundriss der deutschen Wirtschaftsgeschichte, Göttingen 1978

Bundesamt, Statistisches (Hrsg.): Bevölkerung und Wirtschaft 1872 - 1972, Stuttgart 1972

Butterwegge, Christoph: Wohlfahrtsstaat im Wandel. Probleme und Perspektiven der Sozialpolitik, 3. Aufl., Opladen 2001

Castel, Robert: Die Metamorphosen der sozialen Frage. Eine Chronik der Lohnarbeit, Konstanz 2000

Croner, Fritz: Soziologie der Angestellten, Köln 1962

de Swaan, Abram Der sorgende Staat. Wohlfahrt, Gesundheit und Bildung in Europa und den USA der Neuzeit, Frankfurt am Main [Amsterdam] 1993 [1989]

Eichhorst, Werner et al.: Atypische Beschäftigung und Niedriglohnarbeit. Benchmarking Deutschland: Befristete und geringfügige Tätigkeiten, Zeitarbeit und Niedriglohnbereich, Gütersloh 2010

Engelberger, Josef: Tarifautonomie im Deutschen Reich, Berlin 1995

Euchner, Walter: Ideengeschichte des Sozialismus in Deutschland, Teil 1, in: Ideengeschichte des Sozialismus in Deutschland, 2. Aufl., Hrsg.: Grebing, Helga, Wiesbaden 2005, S. 13-354

Faust, Anselm: Arbeitsmarktpolitik in Deutschland im 19. und 20. Jahrhundert: die Arbeitsvermittlung im Wechsel arbeitsmarktpolitischer Strategien, in: Auswanderer-Wanderarbeiter-Gastarbeiter. Bevölkerung, Arbeitsmarkt und Wanderung in Deutschland seit der Mitte des 19. Jahrhunderts, Hrsg.: Bade, Klaus J., Ostfildern 1984, S. 216-253

Fischer, Wolfram et al.: Sozialgeschichtliches Arbeitsbuch. Materialien zur Statistik des Deutschen Bundes 1815-1870, 1, Berlin 1982

Förster, Christian: Die Dimension des Unternehmens, Tübingen 2003

Gall, Lothar (Hrsg.): Stadt und Bürgertum im 19. Jahrhundert, München 1990

Garaudy, Roger: Die Aktualität des Marxschen Denkens, Frankfurt am Main 1969

Grohmann, Heinz: Die Entwicklung eines Bevölkerungsmodells zur Beurteilung der Finanzierung der dynamischen Rente, Berlin 1965

Grumbach, Franz/König, Heinz: Beschäftigung und Löhne der deutschen Industriewirtschaft 1888-1954, in: Weltwirtschaftliches Archiv, Ausgabe 79, 1957,

Hentschel, Volker: Geschichte der deutschen Sozialpolitik 1880-1980, Frankfurt am Main 1983

Herkner, H.: Arbeiterfrage und Sozialreform, Die Arbeiterfrage, 1, 8. Aufl., Berlin/Leipzig 1922

Heuss, Theodor: Robert Bosch. Leben und Leistung, Stuttgart/Tübingen 1946 
Hoffmann, Walter G.: Das Wachstum der deutschen Wirtschaft seit der Mitte des 19. Jahrhunderts, Berlin/Heidelberg/New York 1965

Hueck, Alfred et al.: Lehrbuch des Arbeitsrechts, Band I, 7. Aufl., Berlin 1963

Kocka, Jürgen: Arbeitsverhältnisse und Arbeiterexistenzen, Bonn 1990

Kocka, Jürgen: Mehr Last als Lust. Arbeit und Arbeitsgesellschaft in der europäischen Geschichte, in: Jahrbuch für Wirtschaftsgeschichte, Ausgabe 2, 2005, S. 185-206

Krause, Ferdinand: Die hergebrachten Grundsätze des Berufsbeamtentums. Eine rechtshistorische Analyse, Frankfurt am Main 2008

Kuczynski, Jürgen: Geschichte der Lage der Arbeiter in Deutschland von 1800 bis in die Gegenwart, Bd. 1, Berlin 1947

Kuczynski, Jürgen: Darstellung der Lage der Arbeiter in Deutschland 1789 bis 1849. Geschichte der Arbeiter unter dem Kapitalismus, 1, Berlin 1961

Lampert, Heinz: Krise und Reform des Sozialstaats, Finanzwissenschaftliche Schriften, Bd. 82, Frankfurt am Main 1997

Manes, Alfred: Sozialversicherung, 7. Aufl., Berlin 1928

Marschalck, Peter: Bevölkerungsgeschichte Deutschlands im 19. und 20. Jahrhundert, Frankfurt am Main 1984

Marx, Karl: Pariser Manuskripte, MEW, Ergänzungsband I, Berlin 1956

Meinert, Ruth: Die Entwicklung der Arbeitszeit in der deutschen Industrie 1820-1956, Münster 1958

Merz, Hans-Georg: Beamtentum und Beamtenpolitik in Baden. Studien zu ihrer Geschichte vom Großherzogtum bis in die Anfangsjahre des nationalsozialistischen Herrschaftssystems, Freiburg/München 1985

Molitor, Bruno: Soziale Sicherung, Theorie der Sozialpolitik, 1, München 1987

Mommsen, Hans: Die Gewerkschaften und die Durchsetzung des Sozialstaates in Deutschland, in: Gewerkschaftliche Monatshefte, Ausgabe 2, 1981,

Mückenberger, Ulrich: Arbeitsprozess - Vergesellschaftung - Sozialverfassung, 2. Aufl., Bremen 1985a

Mückenberger, Ulrich: Die Krise des Normalarbeitsverhältnisses. Hat das Arbeitsrecht noch Zukunft?, in: Zeitschrift für Sozialreform, Ausgabe 31, 1985b, S. 415-434, 457-475

Nipperdey, Thomas: Deutsche Geschichte 1866-1918, Band II, Machtstaat vor der Demokratie, 3. Aufl., München 1995

Pierenkemper, Toni: Die Vermarktung von Arbeitskraft in Deutschland: Begriff, Tendenzen, Forschungsprobleme, in: Auswanderer - Wanderarbeiter - Gastarbeiter. Bevölkerung, Arbeitsmarkt und Wanderung in Deutschland seit der Mitte des 19. Jahrhunderts, Hrsg.: Bade, Klaus J., Ostfildern 1984, S. $139-178$

Pierenkemper, Toni: Wirtschaftsgeschichte, München 2005

Pierenkemper, Toni: Beschäftigung und Arbeitsmarkt, in: Moderne Wirtschaftsgeschichte: eine Einführung für Historiker und Ökonomen, Hrsg.: Ambrosius, Gerold et al., München 2006, S. 235-256

Pierenkemper, Toni: Arbeit und Alter in der Geschichte, Otto von Freising-Vorlesungen der Katholischen Universität Eichstätt-Ingolstadt, 25, Wiesbaden 2006a

Pierenkemper, Toni: Beschäftigung und Arbeitsmarkt, in: Moderne Wirtschaftsgeschichte. Eine Einführung für Historiker und Ökonomen, 2. Aufl., Hrsg.: Ambrosius, Gerold et al., München 2006b, S. 235-256 
Pierenkemper, Toni: Der Auf- und Ausbau eines "Normalarbeitsverhältnisses" in Deutschland im 19. und 20. Jahrhundert, in: Geschichte der Arbeitsmärkte, Hrsg.: Walter, Rolf, Stuttgart 2009, S. 77-112

Pierenkemper, Toni/Kendzia, Michael J.: Der vormoderne Allokationsprozess von Arbeit in Deutschland, IZA Discussion Paper Series, 4962,

Pohl, Hans: Sozialgeschichtliche Probleme in der Zeit der Hochindustrialisierung (1870-1914), Paderborn u.a. 1979

Popitz, Heinrich: Der entfremdete Mensch - Zeitkritik und Geschichtsphilosophie des jungen Marx, Basel 1953

Reichelt, Heinrich: Die Arbeitsverhältnisse in einem Berliner Großbetrieb der Maschinenindustrie, Berlin 1906

Rejewski, Harro-Jürgen: Die Pflicht zur politischen Treue im preußischen Beamtenrecht (1850-1918), Berlin 1973

Rimlinger, Gaston V.: Welfare Policy and Industrialization in Europe, America, and Russia, New York 1971

Ritter, Gerhard A.: Über Deutschland. Die Bundesrepublik in der deutschen Geschichte, München 1998

Ritter, Gerhard A./Tenfelde, Klaus: Arbeiter im Deutschen Kaiserreich 1871-1914, Bonn 1992

Rosenstock, Günther: Versicherungstechnische Probleme in der Geschichte der Bismarck'schen Sozialgesetzgebung, Köslin 1934

Rother, Klaus: Die Reichsversicherungsverordnung 1911, Aachen 1994

Rothfels, Hans: Theodor Lohmann und die Kampfjahre der staatlichen Sozialpolitik (1871-1905), Berlin 1927

Rothfels, Hans: Prinzipienfragen der Bismarckschen Sozialpolitik, Königsberg 1929

Rothfels, Hans: Bismarck, Stuttgart 1970

Salais, Robert: La Formation du chômage comme catégorie: le moment des années 30, in: Revue économique, 36. Jg., Ausgabe 2, 1985, S. 321-366

Schildberg-Schroth, Gerhard: Szenen zur Kaiserzeit: Ansichten und Aussichten vom 19. zum 20. Jahrhundert, Dichtung-Wahrheit-Sprache, Bd. 5, Münster/Hamburg/London 2002

Schmoller, Gustav: Begriff und historische Entwicklung des Beamtentums, in: Acta Borussica, Band I, Berlin 1894, S. 15-143

Schmoller, Gustav: Die soziale Frage. Klassenbildung, Arbeiterfrage, Klassenkampf, München/Leipzig 1918

Schmuhl, Hans-Walter: Arbeitsmarktpolitik und Arbeitsverwaltung 1871-2002. Zwischen Fürsorge, Hoheit und Markt, Nürnberg 2003

Schulz, Günther: Die Angestellten seit dem 19. Jahrhundert, Enzyklopädie deutscher Geschichte, Bd. 54, München 2000

Stirken, Angela Der Herr und der Diener. Friedrich Carl von Moser und das Beamtenwesen seiner Zeit, Bonn 1984

Stolleis, Michael: Historische Grundlagen. Sozialpolitik in Deutschland bis 1945, in: Geschichte der Sozialpolitik in Deutschland seit 1945, Band 1, Baden-Baden 2001, S. 199-332

Syrup, Friedrich: 100 Jahre staatliche Sozialpolitik 1839-1939, Stuttgart 1957

Tenfelde, Klaus (Hrsg.): Arbeit und Arbeitserfahrung in der Geschichte, Göttingen 1986

Tesch, Albert: Alfred Krupp, Leipzig 1923 
Ullmann, Peter: Tarifverträge und Tarifpolitik in Deutschland bis 1914. Entstehung und Entwicklung, interessenpolitische Bedingungen und Bedeutung des Tarifvertragswesens für die sozialistischen Gewerkschaften, Frankfurt am Main 1977

Vobruba, Georg: Staatseingriff und Ökonomiefunktion. Der Sozialstaat als Problem für sich selbst, in: Zeitschrift für Soziologie, Ausgabe 7, 1978, S. 130-156

Volkmann, Harald: Carl Zeiss und Ernst Abbe. Ihr Leben und ihr Werk, Abhandlungen und Berichte, 34. Jahrgang, Heft 2, München 1966

Volkmann, Heinrich: Die Arbeiterfrage im preußischen Abgeordnetenhaus 1848 bis 1869, Berlin 1968

von Berlepsch, Hans-Jörg: "Neuer Kurs" im Kaiserreich? Die Arbeiterpolitik des Freiherrn von Berlepsch 1890 bis 1896, Bonn 1987

von Hippel, Wolfgang: Armut, Unterschichten, Randgruppen in der frühen Neuzeit, Enzyklopädie deutscher Geschichte, Bd. 34, München 1995

Wehler, Hans-Ulrich: Das Deutsche Kaiserreich 1871-1918, Deutsche Geschichte 9, 7. Aufl., Göttingen 1994

Wendt, Wolf Rainer: Geschichte der sozialen Arbeit. Die Gesellschaft vor der sozialen Frage, 1, 5. Aufl., Stuttgart 2008

Winkels, Rico S.: Demografischer Wandel: Herausforderungen und Chancen für Personalentwicklung und betriebliche Weiterbildung, Berlin 2007

Wüstner, Kerstin: Arbeitswelt und Organisation. Ein interdisziplinärer Ansatz, 1. Aufl., Wiesbaden 2006

Wunder, Bernd: Die Rekrutierung der Beamtenschaft in Deutschland. Eine historische Betrachtung, Konstanz 1979

Wunder, Bernd: Geschichte der Bürokratie in Deutschland, Neue historische Bibliothek, 281, Frankfurt am Main 1986

Zimmermann, Bénédicte: Arbeitslosigkeit in Deutschland. Zur Entstehung einer sozialen Kategorie, Frankfurt am Main 2006 